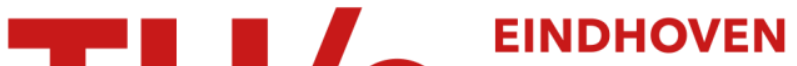 UNIVERSITY OF TECHNOLOGY
}

\section{Effect of particle-size dynamics on properties of dense spongy-particle systems: Approach towards equilibrium}

Citation for published version (APA):

Zakhari, M. E. A., Anderson, P. D., \& Hütter, M. (2017). Effect of particle-size dynamics on properties of dense spongy-particle systems: Approach towards equilibrium. Physical Review E, 96(1), [012604].

https://doi.org/10.1103/PhysRevE.96.012604

DOI:

10.1103/PhysRevE.96.012604

Document status and date:

Published: 18/07/2017

\section{Document Version:}

Accepted manuscript including changes made at the peer-review stage

\section{Please check the document version of this publication:}

- A submitted manuscript is the version of the article upon submission and before peer-review. There can be important differences between the submitted version and the official published version of record. People interested in the research are advised to contact the author for the final version of the publication, or visit the $\mathrm{DOI}$ to the publisher's website.

- The final author version and the galley proof are versions of the publication after peer review.

- The final published version features the final layout of the paper including the volume, issue and page numbers.

Link to publication

\section{General rights}

Copyright and moral rights for the publications made accessible in the public portal are retained by the authors and/or other copyright owners and it is a condition of accessing publications that users recognise and abide by the legal requirements associated with these rights.

- Users may download and print one copy of any publication from the public portal for the purpose of private study or research.

- You may not further distribute the material or use it for any profit-making activity or commercial gain

- You may freely distribute the URL identifying the publication in the public portal.

If the publication is distributed under the terms of Article 25fa of the Dutch Copyright Act, indicated by the "Taverne" license above, please follow below link for the End User Agreement:

www.tue.nl/taverne

Take down policy

If you believe that this document breaches copyright please contact us at:

openaccess@tue.nl

providing details and we will investigate your claim. 


\title{
Effect of particle-size dynamics on properties of dense spongy-particle systems: Approach towards equilibrium
}

\author{
Monica E. A. Zakhari* \\ Polymer Technology, Department of Mechanical Engineering, \\ Eindhoven University of Technology, P.O. Box 513, \\ 5600 MB Eindhoven, The Netherlands and \\ Dutch Polymer Institute (DPI), P.O. Box 902, \\ 5600 AX, Eindhoven, The Netherlands \\ Patrick D. Anderson ${ }^{\dagger}$ and Markus Hütter ${ }^{\ddagger}$ \\ Polymer Technology, Department of Mechanical Engineering, \\ Eindhoven University of Technology, P.O. Box 513, \\ 5600 MB Eindhoven, The Netherlands
}

(Dated: June 27, 2017) 


\begin{abstract}
Open-porous deformable particles, often envisaged as sponges, are ubiquitous in biological and industrial systems (e.g. casein micelles in dairy products and microgels in cosmetics). The rich behavior of these suspensions is owing to the elasticity of the supporting network of the particle, and the viscosity of permeating solvent. Therefore, the rate-dependent size change of these particles depends on their structure, i.e. the permeability. This work aims at investigating the effect of the particle-size dynamics and the underlying particle structure, i.e. the particle permeability, on the transient and long-time behavior of suspensions of spongy particles in the absence of applied deformation, using the dynamic two-scale model developed by Hütter et al. [Faraday Discuss., 158, 407-424 (2012)]. In the high-density limit, the transient behavior is found to be accelerated by the particle-size dynamics, even at average size-changes as small as 1\%. The accelerated dynamics is evidenced by (i) the higher short-time diffusion coefficient as compared to elastic-particle systems and (ii) the accelerated formation of the stable FCC crystal structure. Furthermore, after long times, the particle-size dynamics of spongy particles is shown to result in lower stationary values of the energy and normal stresses as compared to elastic-particle systems. This dependence of the long-time behavior of these systems on the permeability, that essentially is a transport coefficient and hence must not affect the equilibrium properties, confirms that full equilibration has not been reached.
\end{abstract}

PACS numbers: 05.10.-a, 82.70.-y, 83.10.Mj, 83.80.Hj

\footnotetext{
*m.e.a.zakhari@tue.nl

$\dagger$ p.d.anderson@tue.nl

$\ddagger$ m.huetter@tue.nl
} 


\section{INTRODUCTION}

Open-porous, deformable particles are present widely in biological systems, for instance, casein micelles in dairy products, and in several industrial applications, for example, microgel particles in cosmetics and paints [1-3]. Their characteristic sponge-like structure gives rise to elastic behavior due to the elasticity of the supporting network material, while at the same time the particles size-change is hampered at high deformation rates or induced by high packing densities, due to the viscosity of the permeating solvent. Particles with these properties are sometimes referred to as poroelastic particles based on their internal structure $[4,5]$. On the other hand, because of their rate-dependent deformability, spongy-particles are also known to be viscoelastic particles $[6,7]$.

Particle systems, in general, show a transition from a liquid-like to a solid-like behavior as the packing fraction increases [8]. Particles are caged by their neighbors which inhibits their mobility and effectively forming a soft glass. Unlike hard particle systems, soft particles can be packed at densities higher than that for random close-packing, which is 0.64 for monodisperse hard spherical particles. High volume fractions can be reached owing to the different origins of particle softness. The elastic softness allows particles to elastically change their shape, mostly at constant volume, upon contact with their neighbors. In addition, as particles are pressed against each other in a jammed state, viscous solvent is squeezed out of the particle. This type of rate-dependent particle softness enables particles to, over time, change their volume in response to, for instance, steric effects [9]. In the absence of imposed deformation, the competition between the size- and position-dynamics, hence, governs the behavior of such jammed states.

Various modeling efforts have been dedicated to describing the effect of the elastic softness of particles on the emergence of solidity in suspensions of soft particles. The trap models $[10,11]$ are phenomenological models that describe the main features of disordered systems, in particular, aging [11]. An element of these model is trapped in a cage formed by the surrounding elements and, occasionally, this element has enough energy to hop outside this cage due to thermal activation. The Soft Glassy Rheology model (SGR) [12, 13] assumes that hopping can be due to interactions between particles. While these models are used to give insight into the mechanical deformation and aging of these materials, they do not provide any information about the structure. Another limitation of both models is that 
the physical interpretation of the model parameters is not trivial [13], and the latter are not directly related to materials parameters that describe the effective, overall behavior of suspensions.

Particle-based methods, on the other hand, provide more insight about the microscopic dynamics. They form another family of models that are suitable for numerical simulations. They can be categorized into athermal and thermal models. For example, thermal systems consist of particles that are small enough that they are affected by thermal fluctuations. In this case, Brownian forces are relevant. This applies for colloidal suspensions of particles which are a couple of hundred nanometers in size. Although these models have been the basis of the current understanding of the behavior of suspensions of soft particles, they are limited to considering the elasticity of the particle as the only origin of particle softness, for example, using a soft inter-particle potential [14-16]. Spongy-particles, however, can take up (expel) viscous solvent from their interior allowing them to swell (shrink) depending on their permeability, which renders their deformability rate-dependent. Modeling of such particles requires an additional ingredient that is the particle size, to account for the rate-dependent deformability of particles.

This paper aims at studying the effect of the internal structure of spongy particles, in particular the particle permeability, on the equilibrium properties of suspensions of such particles. Understanding the behavior in equilibrium is an essential prerequisite for understanding the behavior under deformation (e.g. shear). The actual approach towards low-energy and the corresponding equilibrium states in dense spongy-particle systems are intricate, which motivates the work in this paper. This is achieved by studying the relaxation of the system from a high-energy initial state through a long-lived intermediate state into a crystalline state, in terms of the transition time and the average local-order parameter, as well as the final overall properties of the system such as energy, stress and diffusion coefficient.

In this paper, the dynamic two-scale model developed by Hütter et al. [17] is applied to dense suspensions of spongy-particles. Based on principals of nonequilibrium thermodynamics, in particular the general equation for the nonequilibrium reversible-irreversible coupling (GENERIC) [18-20], the model accounts, in addition to the elastic origin of particle softness, for the effect of the viscous background fluid by treating the particle-size of each particle as a separate degree of freedom. The developed model is expressed in the form 
of stochastic differential equations [17] suitable for particle-based simulations, specifically Brownian dynamics simulations.

This paper is organized as follows. In Sec. 2, the dynamic two-scale model in the form of stochastic differential equations is revisited and applied to the system of interest by making a choice of the interaction potential. The numerical setup is described in Sec. 3, with a study of the effect of numerical parameters. By making adequate choices of the numerical parameters, the behavior of suspensions of spongy particles is presented for different system densities in Sec. 4. The crystallization of particles is studied in Sec. 5. Finally, the article is concluded with a discussion in Sec. 6.

\section{EXTENDED BROWNIAN DYNAMICS MODEL}

\section{A. Particle interaction}

In this section, a realization of the two-scale model developed in [17] is presented in short. For more details, the reader is referred to [17].

Consider a system of $N$ spherical particles in a Newtonian fluid with shear viscosity $\eta$. Each particle $i$ is described by the position of its center $\boldsymbol{Q}_{i}$ and its radius $R_{i}$. For convenience, a variable $\boldsymbol{\xi}$ of $4 N$-dimension is defined to denote all particle degrees of freedom,

$$
\boldsymbol{\xi}=\left\{\boldsymbol{Q}_{1}, R_{1}, \ldots, \boldsymbol{Q}_{N}, R_{N}\right\}
$$

The distribution function based on the microscopic states $\boldsymbol{\xi}$ is denoted by $p$. In the absence of flow, the Fokker-Planck equation describing the evolution of the distribution function is expressed as

$$
\partial_{t} p=-\nabla_{\boldsymbol{\xi}} \cdot\left(\boldsymbol{\mu} \cdot\left[-\left(\nabla_{\boldsymbol{\xi}} \Phi\right) p-k_{\mathrm{B}} T \nabla_{\boldsymbol{\xi}} p\right]\right)
$$

where $\boldsymbol{\mu}$ is the symmetric and non-negative $4 N \times 4 N$ mobility tensor, $k_{\mathrm{B}}$ is the Boltzmann's constant and $T$ is the absolute temperature. The driving force for $\boldsymbol{\xi}$-dynamics, $-\nabla_{\boldsymbol{\xi}} \Phi$, is derived from the potential energy of the system $\Phi$ for all degrees of freedom. The equilibrium distribution for the microscopic states is given by a Boltzmann distribution [17],

$$
p=\frac{1}{Z} \exp \left(\frac{-\Phi}{k_{\mathrm{B}} T}\right)
$$

where $Z$ is fixed by the normalization condition $\int p d \boldsymbol{\xi}=1$. 
In order to make the model more specific, choices have to be made on the particle interaction potential. Dense suspensions of soft particles are stabilized to prevent aggregation and gelation by, for example, the attractive van der Waals forces [21]. For instance, soft particles in the form of star polymers or polymer-coated particles are stabilized by the outer layer that ensures that the distance between particles is large enough for attractive forces to be negligible $[8,22]$. To that end, the interaction between the particles is assumed in this work to be purely repulsive.

The elasticity of the particles gives rise to these repulsive interactions and is manifested in two different forms. The first form of elastic repulsive interactions is due to the fact that particles are soft and impinging of a particle by its neighbors is possible. The Hertzian potential describes this type of contact energy [23]. The pairwise Hertzian contact interaction potential, between a particle $i$ and its neighbors $j$ 's, is [24, 25]

$$
\Phi_{i j}^{\mathrm{Hz}}(\boldsymbol{\xi})=E R_{\mathrm{eq}}^{3} \tilde{\Phi}_{i j}^{\mathrm{Hz}}
$$

with

$$
\tilde{\Phi}_{i j}^{\mathrm{Hz}}(\boldsymbol{\xi})= \begin{cases}\frac{1}{2\left(1-\nu^{2}\right)}\left(\frac{1}{R_{\mathrm{eq}}^{3}} \sum_{j \neq i}^{N} C h_{i j}^{n} R_{c}^{3-n}+\tilde{k}\right) & \text { if } h_{i j}>0 \\ 0 & \text { otherwise }\end{cases}
$$

where all particles have the same Young's modulus $E$ and Poisson's ratio $\nu$. The freestanding equilibrium particle-size is the same for all particles and is denoted by $R_{\text {eq }}$. The symbol $(\tilde{)})$ denotes the nondimensionalized $(\cdot)$. The contact size, $R_{c}$, is defined as $R_{c}=$ $\left(\frac{1}{R_{i}}+\frac{1}{R_{j}}\right)^{-1}$ where $R_{i}$ and $R_{j}$ are the current particle radii of particles $i$ and $j$, respectively. The overlap between particles is defined as

$$
h_{i j}=R_{i}+R_{j}-\left|\boldsymbol{Q}_{i j}\right|
$$

where the magnitude of the vector connecting particle $i$ and $j$ is $\left|\boldsymbol{Q}_{i j}\right|=\left|\boldsymbol{Q}_{j}-\boldsymbol{Q}_{i}\right|$. The dimensionless constants $C$ and $n$ in Eq. (5) depend on the degree of overlap between a pair of particles, $h_{i j}$. The values of $C, n$ and $\tilde{k}$ used throughout this work can be found in Appendix A.

The Hertzian potential interaction describes the force developed when the particle centers approach closer than the sum of their radii because the particles can deform. This potential 
gives a non-zero contribution only when particles are overlapping, while it vanishes when particles are not impinging, such as in the case of dilute systems. In the dilute-limit, a particle with size that is different from its equilibrium size $R_{\text {eq }}$ will have a finite stored elastic energy [26]. Minimization of this energy drives the particle to recover its equilibrium size. In this work, we account for the stored elastic energy in the total potential. The stored elastic energy, in this case, is

$$
\Phi_{i}^{\mathrm{vol}}(\boldsymbol{\xi})=E R_{\mathrm{eq}}^{3} \tilde{\Phi}_{i}^{\mathrm{vol}},
$$

with

$$
\tilde{\Phi}_{i}^{\mathrm{vol}}=\frac{2 \pi}{9(1-2 \nu)}\left(\frac{V_{i}-V_{\mathrm{eq}}}{V_{\mathrm{eq}}}\right)^{2},
$$

where $V_{i}$ is the current volume of particle $i, V_{\text {eq }}$ is its volume based on the equilibrium size and $\left(V_{i}-V_{\text {eq }}\right) / V_{\text {eq }}$ is the volumetric strain. The total potential energy is defined, based on (4) and (7), as

$$
\Phi(\boldsymbol{\xi})=\sum_{i \neq j} \Phi_{i j}^{\mathrm{Hz}}(\boldsymbol{\xi})+\sum_{i} \Phi_{i}^{\mathrm{vol}}(\boldsymbol{\xi})=E R_{\mathrm{eq}}^{3} \tilde{\Phi}(\boldsymbol{\xi})
$$

In practice, even if particles are not overlapping, an isolated particle strives to attain its equilibrium size, whereas every other (deviating) particle size is energetically penalized. This is exactly what is accounted for by the additive contribution in Eq. (9) as opposed to a total potential that consists of only Hertzian-contact contributions with a density-dependent prefactor. A density-dependent Hertzian potential automatically implies that this potential is only active if particles are impinging, while it vanishes in the dilute limit (i.e., for nonoverlapping particles). In contrast, Eq. (9) ensures that in the dilute limit a particle strives towards its equilibrium size based on the minimization of $\tilde{\Phi}=\tilde{\Phi}^{\mathrm{vol}}$. In addition, it is mentioned for completeness that the density dependence of the particle stiffness in the Hertzian interaction is incorporated in our approach in an effective way by using piece-wise different expressions for the potential Eq. (5) (see Appendix A). 


\section{B. Particle dynamics}

Equation (2) can be expressed in the form of stochastic differential equations using the Itô-interpretation [27]. The position and size dynamics are, respectively, defined as

$$
\begin{aligned}
d \boldsymbol{Q}_{i} & =\sum_{k} \boldsymbol{\mu}_{\boldsymbol{Q}_{i} \boldsymbol{Q}_{k}} \cdot\left(-\nabla_{\boldsymbol{Q}_{k}} \Phi\right) d t+\sum_{k} \boldsymbol{\mu}_{\boldsymbol{Q}_{i} R_{k}}\left(-\partial_{R_{k}} \Phi\right) d t \\
& +k_{\mathrm{B}} T\left[\nabla_{\boldsymbol{\xi}} \cdot \boldsymbol{\mu}\right]_{\boldsymbol{Q}_{i}} d t+\left[\boldsymbol{B} d \boldsymbol{W}_{t}\right]_{\boldsymbol{Q}_{i}} \\
d R_{i} & =\sum_{k} \boldsymbol{\mu}_{R_{i} \boldsymbol{Q}_{k}} \cdot\left(-\nabla_{\boldsymbol{Q}_{k}} \Phi\right) d t+\sum_{k} \mu_{R_{i} R_{k}}\left(-\partial_{R_{k}} \Phi\right) d t \\
& +k_{\mathrm{B}} T\left[\nabla_{\boldsymbol{\xi}} \cdot \boldsymbol{\mu}\right]_{R_{i}} d t+\left[\boldsymbol{B} d \boldsymbol{W}_{t}\right]_{R_{i}}
\end{aligned}
$$

where $\boldsymbol{B}$ satisfies the fluctuation-dissipation relation $\boldsymbol{B} \cdot \boldsymbol{B}^{T}=2 k_{\mathrm{B}} T \boldsymbol{\mu}$. The driving forces for position-change and size-change are $-\nabla_{\boldsymbol{Q}_{i}} \Phi$ and $-\partial_{R_{i}} \Phi$, respectively.

For simplicity and in order to focus on the effect of the permeability, many-particle hydrodynamic interactions are neglected, which implies that $\boldsymbol{\mu}_{\boldsymbol{Q}_{i} \boldsymbol{Q}_{k}}$ is non-zero only if $i=k$, specifically [28]

$$
\boldsymbol{\mu}_{\boldsymbol{Q}_{i} \boldsymbol{Q}_{k}}=\zeta_{Q_{i}}^{-1} \delta_{i k} \boldsymbol{I}, \text { with } \zeta_{Q_{i}}=6 \pi \eta R_{i}
$$

where $\boldsymbol{I}$ is the second rank identity tensor, and $\zeta_{Q_{i}}$ is the single-particle friction coefficient. Furthermore, it is assumed that the size-dynamics of different particles are not coupled hydrodynamically, which is a reasonable assumption since the fluid is assumed to be (nearly) incompressible [17]. Therefore, one obtains $\mu_{R_{i} R_{k \neq i}}=0$, and the single-particle mobility component can be expressed as [17]

$$
\mu_{R_{i} R_{k}}=\zeta_{R_{i}}^{-1} \delta_{i k}, \text { with } \zeta_{R_{i}}=\frac{12 \pi \eta R_{i}^{4}}{R_{\mathrm{eq}}^{3} \chi}
$$

where $\chi$ is a dimensionless quantity which depends on the permeability of the particle. Using the theory of poroelasticity [29], it can be shown that

$$
\chi=\frac{\pi^{2} \kappa}{R_{0}^{2}},
$$

where $\kappa$ is the particle permeability and $R_{0}$ is the initial particle size which is equal to $R_{\text {eq }}$ in this work (see Sec. III A). Non-zero mobility components $\boldsymbol{\mu}_{\boldsymbol{Q}_{i} R_{k}}$ and $\boldsymbol{\mu}_{R_{i} \boldsymbol{Q}_{k}}$ imply that there is dynamic cross-coupling between size and position. For the current work, we assume that there is no cross-coupling, that is $\boldsymbol{\mu}_{\boldsymbol{Q}_{i} R_{k}}=\mathbf{0}$ and $\boldsymbol{\mu}_{R_{i} \boldsymbol{Q}_{k}}=\mathbf{0}$. 
Based on the above-mentioned assumptions, equations (10a) and (10b) are simplified to

$$
\begin{aligned}
d \boldsymbol{Q}_{i} & =\zeta_{Q_{i}}^{-1} E R_{\mathrm{eq}}^{3}\left(-\nabla_{\boldsymbol{Q}_{i}} \tilde{\Phi}\right) d t+\sqrt{2 k_{\mathrm{B}} T \zeta_{Q_{i}}^{-1}}\left[d \boldsymbol{W}_{t}\right]_{\boldsymbol{Q}_{i}}, \\
d R_{i} & =\zeta_{R_{i}}^{-1} E R_{\mathrm{eq}}^{3}\left(-\partial_{R_{i}} \tilde{\Phi}\right) d t+k_{\mathrm{B}} T\left(\partial_{R_{i}} \zeta_{R_{i}}^{-1}\right) d t+\sqrt{2 k_{\mathrm{B}} T \zeta_{R_{i}}^{-1}}\left[d W_{t}\right]_{R_{i}} .
\end{aligned}
$$

These equations can be non-dimensionalized if one scales all involved length scales by $R_{\text {eq }}$,

$$
\begin{aligned}
& d \tilde{\boldsymbol{Q}}_{i}=\zeta_{Q_{i}}^{-1} E R_{\mathrm{eq}}\left(-\boldsymbol{\nabla}_{\tilde{Q}_{i}} \tilde{\Phi}\right) d t+\sqrt{2 k_{\mathrm{B}} T \zeta_{Q_{i}}^{-1} R_{\mathrm{eq}}^{-2}}\left[d \boldsymbol{W}_{t}\right]_{\boldsymbol{Q}_{i}}, \\
& d \tilde{R}_{i}=\zeta_{R_{i}}^{-1} E R_{\mathrm{eq}}\left(-\partial_{\tilde{R}_{i}} \tilde{\Phi}\right) d t+k_{\mathrm{B}} T R_{\mathrm{eq}}^{-2}\left(\partial_{\tilde{R}_{i}} \zeta_{R_{i}}^{-1}\right) d t+\sqrt{2 k_{\mathrm{B}} T \zeta_{R_{i}}^{-1} R_{\mathrm{eq}}^{-2}}\left[d W_{t}\right]_{R_{i}} .
\end{aligned}
$$

\section{Time scales and dimensionless numbers}

Equations (15) are expressed in terms two energy scales: the elastic interaction energy, $E R_{\text {eq }}^{3}$, and the thermal energy, $k_{\mathrm{B}} T$. The ratio between these energy scales defines the first dimensionless parameter describing the elastic nature of the particle softness, and is defined as

$$
S^{*}=\frac{k_{\mathrm{B}} T}{E R_{\mathrm{eq}}^{3}} .
$$

This parameter is similar to the softness defined by Ikeda et al. in [14] in terms of its physical interpretation, but we use a different form of the interaction energy.

The effect of the suspending fluid is described by the second dimensionless parameter, that is defined as the ratio of mobilities in the size dynamics and position dynamics, respectively,

$$
\zeta^{*}=\left.\frac{\zeta_{R_{i}}^{-1}}{\zeta_{Q_{i}}^{-1}}\right|_{R_{i}=R_{\mathrm{eq}}}=\frac{\chi}{2} .
$$

It follows from (13) that this parameter is based on the initial size of the particle as well as its permeability. Based on the above dimensionless parameters, several familiar systems can be recovered. For instance, hard particle systems are recovered by setting $S^{*}=0$ and $\zeta^{*}=0$. Elastic (impermeable) particles are obtained for a finite value of $S^{*}$ and $\zeta^{*}=0$, while non-zero $\left(S^{*}, \zeta^{*}\right)$ describe soft porous particles.

The energy scales define different time scales for the position and size dynamics, respectively. The interaction energy is dissipated through viscous damping over a time scale, for position change and for volume change,

$$
\tau_{Q_{i}}=\frac{\zeta_{Q_{i}}}{E R_{\mathrm{eq}}},
$$


and

$$
\tau_{R_{i}}=\frac{\zeta_{R_{i}}}{E R_{\mathrm{eq}}}
$$

respectively. The Brownian time scale is

$$
\tau_{Q_{i}}^{\mathrm{Br}}=\frac{\zeta_{Q_{i}} R_{\mathrm{eq}}^{2}}{k_{\mathrm{B}} T},
$$

which is the time a particle takes to diffuse a distance equivalent to its radius, while a particle relaxes its size under the influence of thermal fluctuations over a time scale

$$
\tau_{R_{i}}^{\mathrm{Br}}=\frac{\zeta_{R_{i}} R_{\mathrm{eq}}^{2}}{k_{\mathrm{B}} T} .
$$

Equation (15) can be expressed, in terms of the previously described time scales, as

$$
\begin{aligned}
& d \tilde{\boldsymbol{Q}}_{i}=\left(-\boldsymbol{\nabla}_{\tilde{\boldsymbol{Q}}_{i}} \tilde{\Phi}\right) \frac{d t}{\tau_{Q_{i}}}+\sqrt{2} \frac{\left[d \boldsymbol{W}_{t}\right]_{\boldsymbol{Q}_{i}}}{\sqrt{\tau_{Q_{i}}^{\mathrm{Br}}}}, \\
& d \tilde{R}_{i}=\left(-\partial_{\tilde{R}_{i}} \tilde{\Phi}\right) \frac{d t}{\tau_{R_{i}}}+\left(\partial_{\tilde{R}_{i}} \ln \zeta_{R_{i}}^{-1}\right) \frac{d t}{\tau_{R_{i}}^{\mathrm{Br}}}+\sqrt{2} \frac{\left[d \boldsymbol{W}_{t}\right]_{R_{i}}}{\sqrt{\tau_{R_{i}}^{\mathrm{Br}}}} .
\end{aligned}
$$

\section{NUMERICAL SIMULATIONS}

\section{A. Temporal discretization and simulation parameters}

Equations (22) are used to model a system consisting of $N$ particles in a cubic box. The simulation box is subjected to Lees-Edwards periodic boundary conditions [30]. Monodisperse particles, with initial size $R_{0}=R_{\text {eq }}$, are initially placed on a Simple Cubic (SC) configuration (unless otherwise specified) in the simulation box, suspended in a solvent of viscosity $\eta$. By changing the dimensions of the simulation box, the density of the system is modified while keeping the number of particles fixed. The parameters used throughout this paper are listed in Table I. The characteristic time scales, based on physical values in Table I, are shown in Table II. A forward-Euler integration scheme is used to solve equation (15) [31]. The time step used for the integration scheme, $\Delta t$, should be small enough to capture the fastest dynamics. Table II shows that the smallest time scale is $\tau_{Q}$. The time step is set to be two orders of magnitude smaller, that is $\Delta t=3.5 \cdot 10^{-6} \mathrm{~s}$.

To provide an indication of typical values for the dimensional parameters, some examples

can be found in literature. For instance, the softness dimensionless number $S^{*}$ is estimated 
TABLE I: Model parameters.

\begin{tabular}{lll} 
Parameter & \multicolumn{2}{l}{ Symbol Physical value } \\
\hline Equilibrium particle radius & $R_{\mathrm{eq}}$ & $2.5 \cdot 10^{-7} \mathrm{~m}$ \\
Solvent shear viscosity & $\eta$ & $10^{-3} \mathrm{~Pa} \mathrm{~s}$ \\
Poisson's ratio & $\nu$ & 0.4 \\
Young's modulus & $E$ & $100 \mathrm{~Pa}$ \\
Temperature & $T$ & $293 \mathrm{~K}$ \\
Number of particles & $N$ & $12^{3} \mathrm{a}$ \\
Softness, dimensionless & $S^{*}$ & $2.5 \cdot 10^{-3}$ \\
Permeability, dimensionless $\zeta^{*}$ & $\{0,0.5,5\} \cdot 10^{-3}$ \\
\hline
\end{tabular}

${ }^{\text {a }}$ Additional simulations were performed for $N=22^{3}$ with no significant effect on the overall behavior (see Appendix B).

TABLE II: Characteristic time scales based on the model parameters in Table I, using $R_{i}=R_{\text {eq }}$. Values for $\tau_{R}$ and $\tau_{R}^{\mathrm{Br}}$ are based on $\zeta^{*}$ in Table I.

\begin{tabular}{ll}
\multicolumn{2}{l}{ Time scale Physical value $[\mathrm{s}]$} \\
\hline$\tau_{Q}$ & $1.88 \cdot 10^{-4}$ \\
$\tau_{Q}^{\mathrm{Br}}$ & $7.28 \cdot 10^{-2}$ \\
$\tau_{R}$ & $\left\{\infty, 3.77 \cdot 10^{-1}, 3.77 \cdot 10^{-2}\right\}$ \\
$\tau_{R}^{\mathrm{Br}}$ & $\{\infty, 145.6,14.56\}$ \\
\hline
\end{tabular}

to be $10^{-7}$ for oil-in-water emulsions and between $10^{-4}-10^{-5}$ for polyacrylamide particles (e.g. PNIPAM) $[8,14]$. Furthermore, to get an estimate for the dimensionless permeability $\zeta^{*}$, the permeability of polyacrylamide particles is considered (oil-in-water emulsions are impermeable), which is estimated to be higher than $10^{-17} \mathrm{~m}^{2}$ depending on the cross-link density [32]. For sub-micron sized polyacrylamide particles, one finds that $\zeta^{*}>10^{-3}$ [14]. The model parameters in Table I correspond to systems consisting of particles with permeability, and hence $\zeta^{*}$, similar to the widely-used polyacrylamide particles in water [14, 32]. On the other hand, for the purpose of visualizing the particle-size change, the particle elastic properties, and consequently $S^{*}$, in Table I are one order of magnitude softer than those 
commonly reported for polyacrylamide particles. The elastic properties in Table I are, however, similar to those of systems used to mimic biological systems, for instance alginate hydrogels used for cell encapsulating scaffolds [33], vinylpyridine (VP) microgels used in applications where pH-sensitivity is important [34], and polyisocyanides (PIC) hydrogels used as a biomimetic system [35]. It is noteworthy that, while $S^{*}$ given by (16) is convenient for scaling the evolution equations, it is more physical to express $S^{*}$ in terms of elastic energy for volume change, that is approximately equal to $k_{\mathrm{B}} T /\left(3 E R_{\mathrm{eq}}^{2} \Delta R\right)$ where $\Delta R$ is the radius change with respect to $R_{\text {eq }}$. One finds that, for a radius change of approximately $8 \cdot 10^{-4} R_{\text {eq }}$, the elastic energy for volume change is equal to the thermal energy $k_{\mathrm{B}} T$.

At high number densities and no external flow, particle positions and sizes change as a result of interactions and Brownian forces in order to reduce total energy of the system and to balance the forces acting on each particle. The evolution of the system over time is measured in terms of the energy per particle.

\section{B. Evolution of energy}

As a prototypical example, a system with number density $n=1.17 \cdot 10^{19} \mathrm{~m}^{-3}$ is simulated. In this example, particles are initially placed randomly in the simulation box. The volume fraction can be calculated, based on the equilibrium size, as

$$
\varphi_{\text {free }}=\frac{4}{3} \pi R_{\mathrm{eq}}^{3} n
$$

That yields a volume fraction of 0.766 for this example. The particles used are porous with $\zeta^{*}=0.005$. The simulation time is chosen to be large enough to allow the particles to travel longer distances than their own diameter. To obtain statistically relevant data, simulations long compared to the Brownian time scale $\tau_{Q}^{\mathrm{Br}}$ are performed,

$$
\tau_{\text {sim }}=28 s \approx 384 \tau_{Q}^{\mathrm{Br}},
$$

where $\tau_{Q}^{\mathrm{Br}}=7.28 \cdot 10^{-2} \mathrm{~s}$, rather than performing several shorter simulations of independent systems.

Figure 1 shows the evolution of the energy per particle for this system. Initially, the energy decreases slowly over a period of approximately $45.3 \tau_{Q}^{\mathrm{Br}}$. This is followed by a drop in energy of a value of $\Delta \Phi \approx 0.75 k_{\mathrm{B}} T$ per particle. This energy drop is found to be related to 
a transition from a disordered to an ordered state. This is confirmed by the snapshots of the particle configuration in the simulation box at the beginning and the end of the simulation shown in Fig. 2 and will be discussed in more detail in Section V. Structural ordering was observed in hard- and soft-particle systems. This phenomena depends primarily on the particle interactions [36-38] and the size polydispersity [8,39]. Ordering occurs more readily when repulsive interactions are dominant while polydispersity inhibits crystal formation [8].

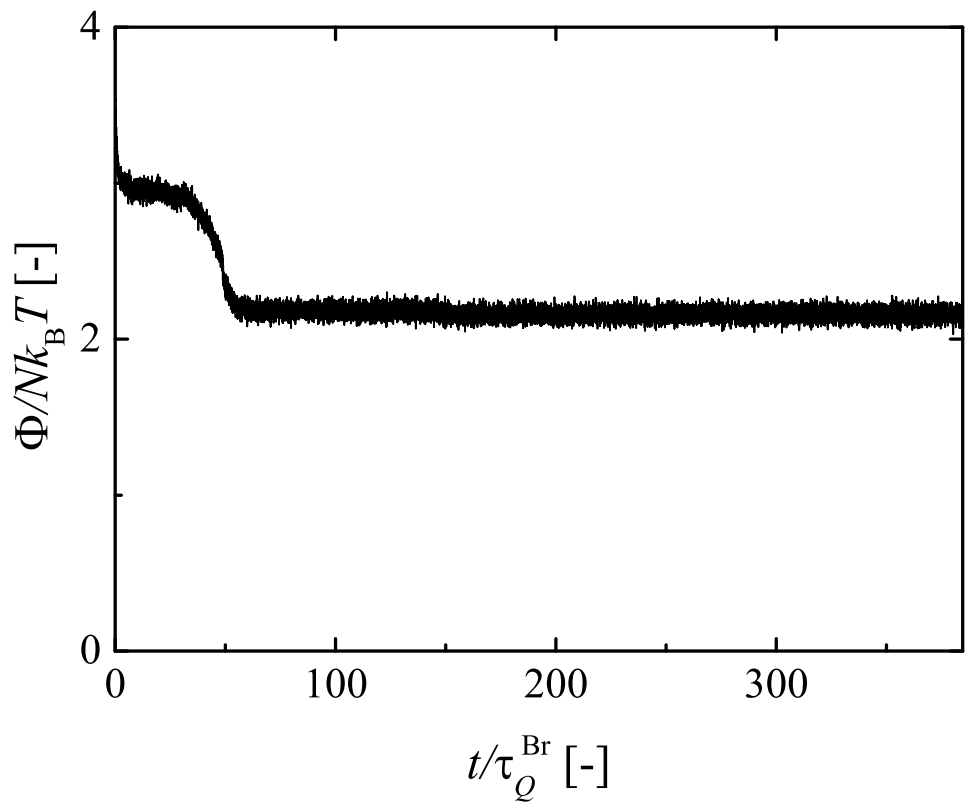

FIG. 1: Evolution of energy per particle for a system with particle with $\zeta^{*}=0.005$ and $S^{*}=2.5 \cdot 10^{-3}$. The volume fraction of the system is $\varphi_{\text {free }}=0.766\left(n=1.17 \cdot 10^{19} \mathrm{~m}^{-3}\right)$.

The time-dependent response of the material, as seen in Fig. 1, implies that the simulation time should be long enough to allow particles to find the lower energy state. The transition time involved in this process occurs at time scales significantly larger than the Brownian time scale (see Table II). This long transition-time implies that the disordered state at intermediate energy, which occurs at $5 \lesssim t / \tau_{Q}^{\mathrm{Br}} \lesssim 30$, is considered a long-lived glassy state. Intuitively, one expects that this slow dynamics is accompanied by large energy barriers. The system might be trapped in a metastable state and particles do not have enough energy to cross a high energy barrier to reach a lower energy. However, such energy barriers were not observed for the range of parameters studied. This suggests that structural ordering occurs spontaneously and therefore small differences might have a significant effect on the subsequent relaxation, which would imply a substantial dependence on the sample history. 

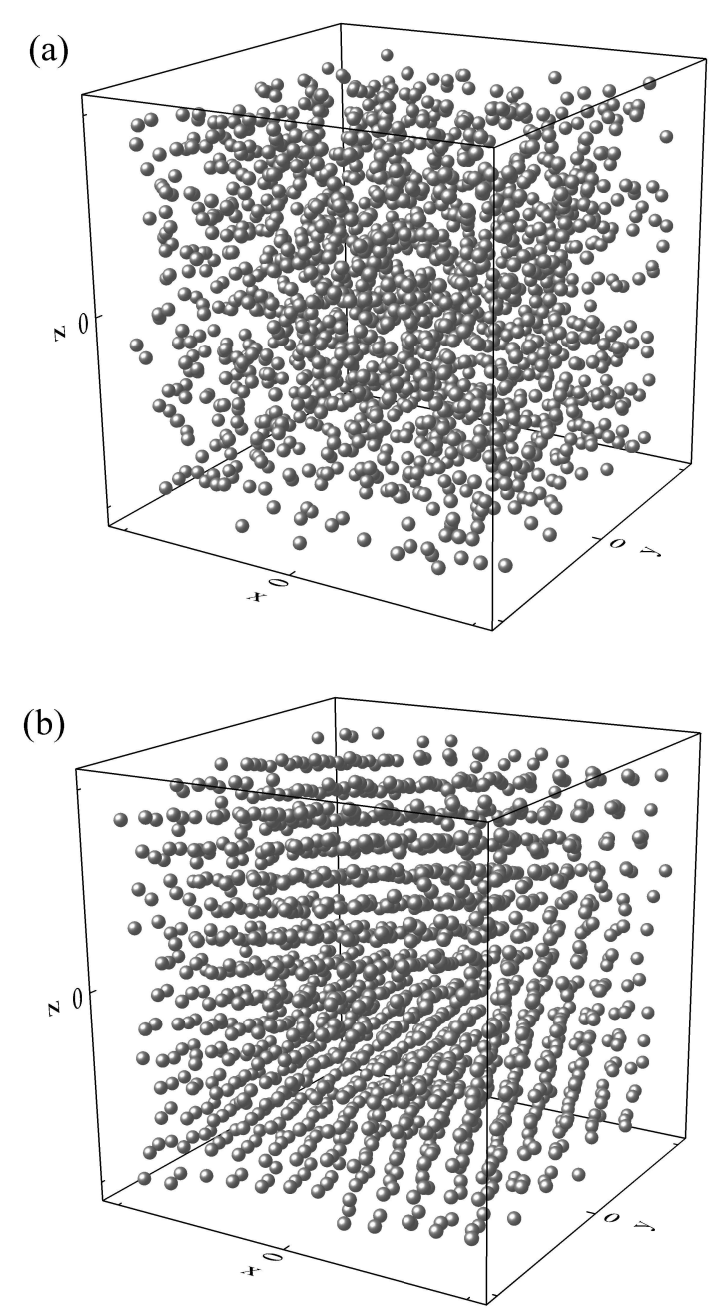

FIG. 2: Particle configuration at (a) $t / \tau_{Q}^{\mathrm{Br}}=0$ and (b) $t / \tau_{Q}^{\mathrm{Br}}=384.6$. Particle coordinates are scaled by the simulation box length and the particle size is reduced for better visualization.

For the systems examined in this numerical study, the history represents the initial state of the system as well as the pathway followed by the particles in the relaxation process. History can be removed when deformation is applied [40], however, in this work we are interested in equilibrium properties where there is no applied deformation. In the case of no applied deformation, care should be taken when drawing conclusions about the absolute behavior of such systems as it might be a direct effect of its history. To this end and in order to isolate the effect of the history, the effect of the numerical parameters on the simulation results is studied in detail next. 


\section{Effect of numerical parameters}

This section is dedicated to analyze the effect of numerical parameters on the behavior of the suspension at high volume fractions. As the density of the system increases, particles require more time to find a configuration that minimizes the total energy of the system, i.e. the glassy state lives longer. Dynamics are drastically slowed and the material increasingly remembers its history. The history-dependent behavior dictates a careful study of the simulation history that we identify as: the initial configuration, and the realization of the noise (i.e. the last terms in Eq. (22a) and (22b)). In order to examine this slow relaxation towards equilibrium through or from intermediate long-lived states, two types of well-defined initial (high energy) configurations are generated by (i) randomly placing particle centers in the simulation box, and by (ii) placing the particle on a simple cubic lattice. In both cases, a long-lived (glassy) state develops relatively rapidly due to the dynamics of the system (see Fig. 1, $5 \lesssim t / \tau_{Q}^{\mathrm{Br}} \lesssim 30$ ), which in turn eventually evolves into a crystalline low-energy state (see Fig. 1, $t / \tau_{Q}^{\mathrm{Br}} \gtrsim 60$ ). The first relatively rapid transition from the initial state is not of interest in this paper, but the main focus is rather the transition from the long-lived intermediate state towards the low-energy state. The realization of noise for the thermal fluctuation terms in Eq. (22) can be changed by varying the random number seed.

Two sets of simulations are performed for suspensions with different particle volume fractions. The first set of simulations is done for five different random initial configurations while fixing the realization of the noise. In this case, the initial particle size for all particles is the equilibrium size. In the second set of simulations, the initial configuration is fixed by placing particles in a SC configuration at different realizations of the noise. The initial particle radius of porous particles in both sets of simulation is set to the equilibrium size. However, the initial size of impermeable particles is drawn from a Gaussian distribution around the equilibrium size. The reason for this is the following. Size dynamics allows for the radii of permeable particles to conform to a certain, evolving distribution. At equilibrium, this distribution is the Boltzmann distribution given by Eq. (3). If one considers the volumetric energy contribution only, equation (3) is reduced to

$$
p=\frac{1}{Z} \exp \left(\frac{-\Phi^{\mathrm{vol}}}{k_{\mathrm{B}} T}\right) \text {. }
$$

At equilibrium, the energy $\Phi_{i}^{\mathrm{vol}}$ and the driving force $-\partial_{R} \Phi_{i}^{\mathrm{vol}}$ vanish up to noise contributions. Particle-size fluctuations around $R_{\text {eq }}$ result in an average contribution to the energy 
of $k_{\mathrm{B}} T / 2$, based on the equipartition theorem. To account for this finite energy contribution in the simulations with impermeable particles, a Monte-Carlo algorithm is used to generate uncorrelated initial particle radii. The Monte-Carlo algorithm generates radii with a Boltzmann distribution around $R_{\text {eq }}$ with respect to the volumetric energy (Eq. (7)). So doing, the simulations of permeable and impermeable particles can be compared more easily.

The effect of the initial configuration and the realization of the noise at different volume fractions are studied in terms of the energy per particle (Fig. 3) and the transition time (Fig. 4). The energy per particle is calculated based on the average of energy over a period of $t_{\text {end }}-t_{\text {start }}=96.2 \tau_{Q}^{\mathrm{Br}}$, with $t_{\text {start }}>\tau_{\mathrm{t}}$. The transition time, $\tau_{\mathrm{t}}$, is defined as the time at which the energy of the system is lowered as particles form an ordered phase. It is obtained by fitting the energy-time curve with a hyperbolic tangent function with the inflection point denoting the transition time. It is noteworthy that, no overlap criteria for placing the particles randomly in the simulation box is imposed, the initial overlap and contact force can be large for some configurations. Porous particles can change their size to reduce these large forces. This is not the case for impermeable particles and in order to resolve the dynamics, in this case, the time step needs to be reduced significantly. Therefore the computational expense may become prohibitive. This is why results for $\zeta^{*}=0$ are not shown in Fig. 3b and Fig. 4b.

Figure 3 shows that the energy values are not affected by the choice of initial configuration or realization of the noise. This means that at longer times the history has already been removed. In other words, the system is not quenched and its history does not influence the behavior of the material, as far as the energy value is concerned. However, this does not apply for the transition time, Fig. 4. Initially, crystallization is slow, i.e. longer transition time, because of small saturation compared to the freezing point, that is the first density at which a crystal structure occurs. Based on our simulations, the freezing point is a value between $1.1 \cdot 10^{19}<n<1.13 \cdot 10^{19} \mathrm{~m}^{-3}$, that is $0.72<\varphi_{\text {free }}<0.74$. Upon increasing the system density, the effective particle diffusion coefficient is reduced and particles require more time for structural rearrangement, hence the increased $\tau_{\mathrm{t}}$ on average (see Sec. IV B). The behavior of the transition time agrees qualitatively with the work of Pusey et al. [41] for hard particle suspensions. Furthermore, the transition time is highly affected by the choice of initial condition and the realization of the noise (see Fig. 4). The spread of data in Fig. 4a and Fig. 4b increases with increasing system density. This has implications on 

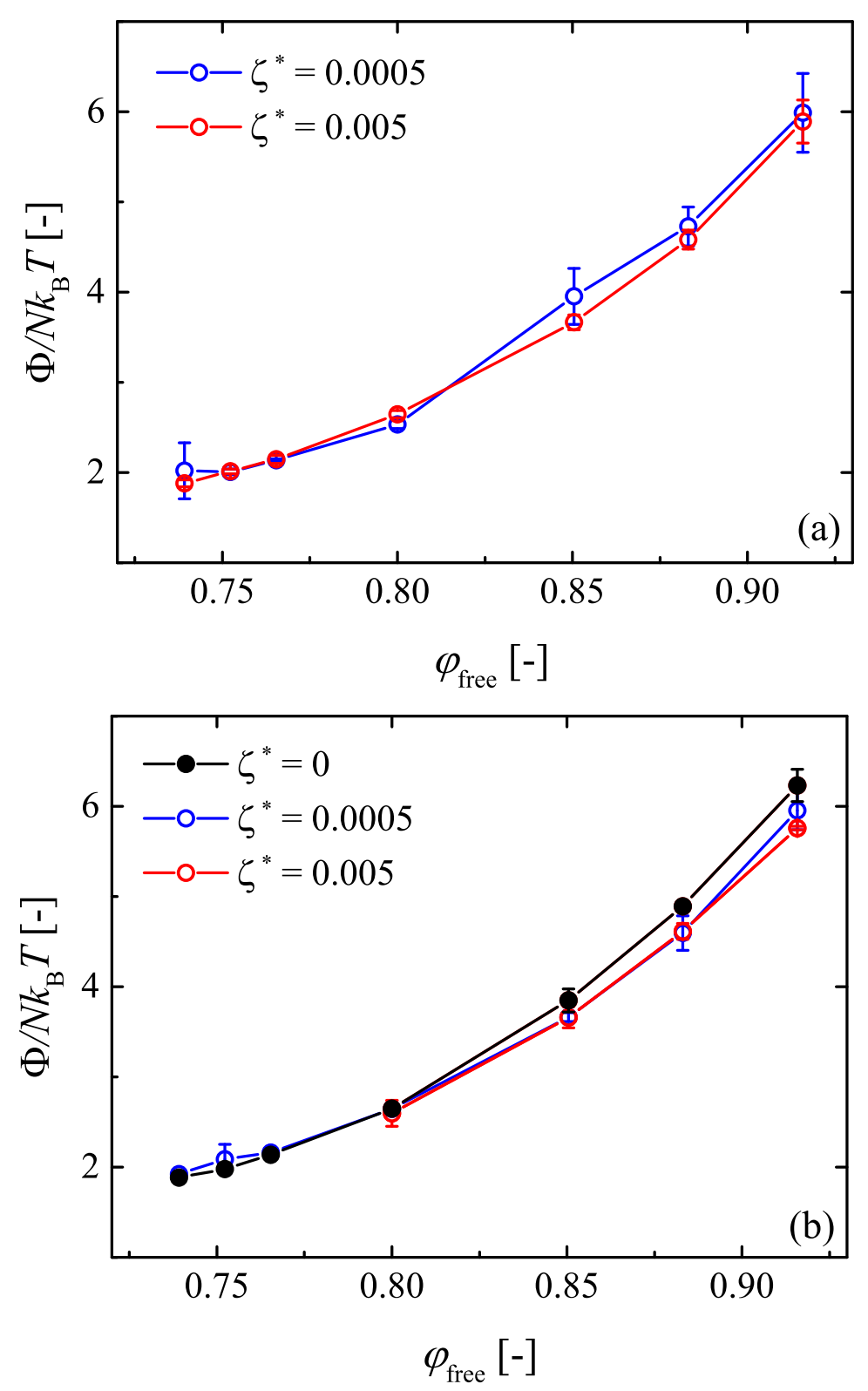

FIG. 3: Energy per particle for systems with different $\zeta^{*}$ and $n$ averaged over (a) 5 different initial configurations and certain realization of the noise, (b) 5 different realizations of the noise and a fixed initial configuration. The standard deviation of the data is shown by the error bars.

the simulation time. It should be chosen long enough for the glassy state to elapse and to allow the particles to find a lower energy configuration.

Based on the results shown in this section, one can draw the following conclusions. The simulation time should allow for the collective motion of the particles in the entire system to 

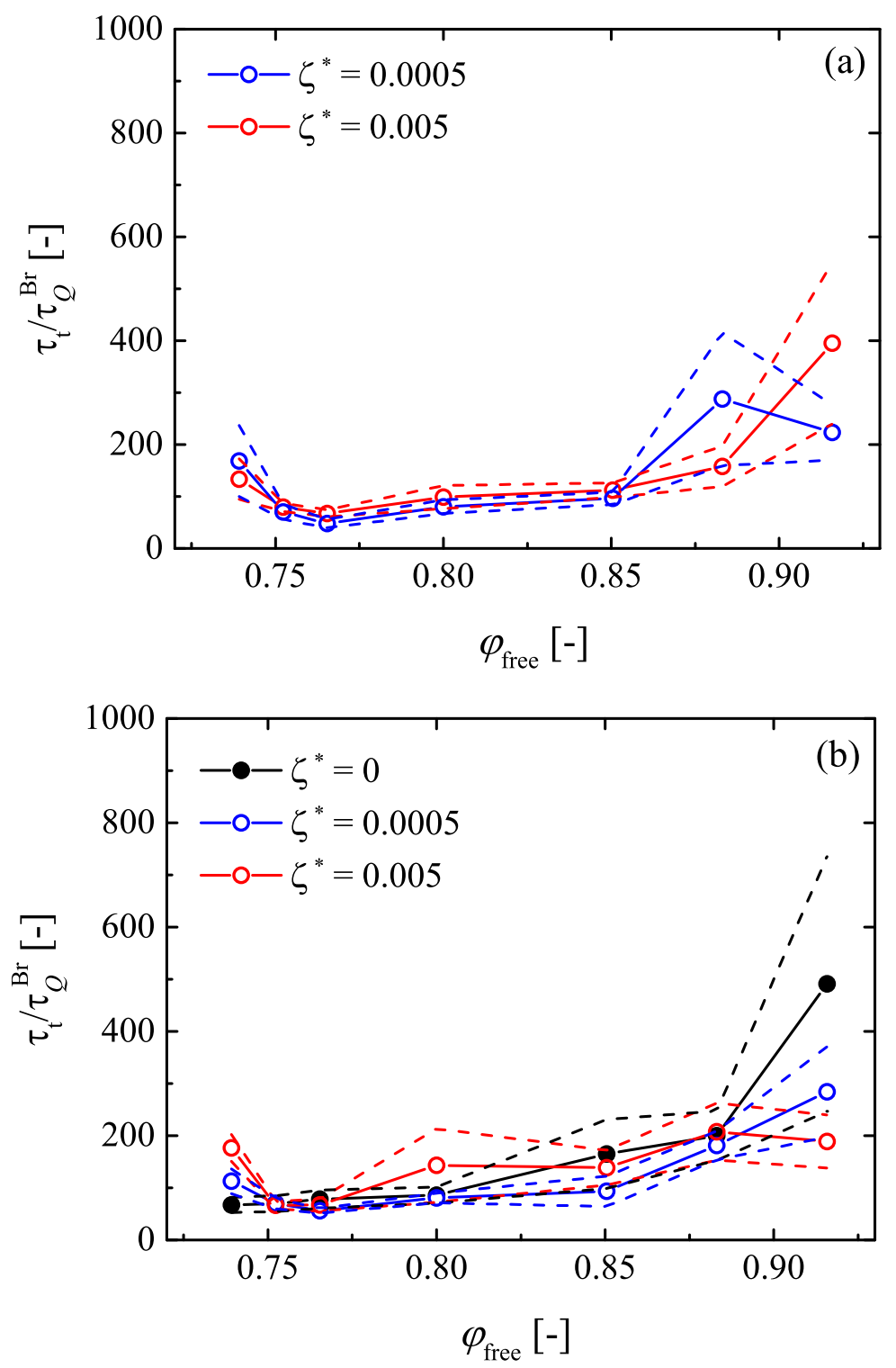

FIG. 4: Transition time $\tau_{\mathrm{t}}$ for systems with different $\zeta^{*}$ and $n$ averaged over (a) 5 different initial configurations and a certain realization of the noise, (b) 5 different realizations of the noise and a fixed initial configuration. The spread in data based on the standard error is shown by dashed lines.

find a lower-energy state. The time scale involved in this process is complicated to estimate beforehand. The reason for this is that, first, it happens on time scales significantly larger than the Brownian time scale $\tau_{Q}^{\mathrm{Br}}$. This transition-time scale grows as the density of the system increases. Secondly, we show that this transition-time scale depends on the system history (initial configuration), and pathway taken by the particles (the realization of the 
noise). Here, we relate this time scale to the transition time for structural ordering, $\tau_{\mathrm{t}}$, see Fig. 2. As a result, the transition time imposes an additional condition on the simulation time, that is the simulation time should be larger than the transition time to measure material properties that are independent of the system history. Based on these arguments, the simulation time used in the remainder of the paper is

$$
\tau_{\text {sim }} \geq 2 \tau_{\mathrm{t}}
$$

It is also found that impermeable particles have higher energy per particle than porous particles (see Fig. 3b). In addition, they undergo a transition to an ordered state on longer time scales, on average, than porous particles in particular at higher densities (see Fig. 4b). In contrast to comparing porous and non-porous particles, let us compare porous-particle systems with different permeability, i.e. $\zeta^{*} \neq 0$. The effect of the value of permeability of porous particles on the energy and transition time is unclear as can be seen in Fig. 3 and 4, respectively. These observations will be studied further in the following sections. Henceforth, in order to compare results with systems consisting of impermeable particles, we focus on systems with fixed simple-cubic initial configuration and different realizations of the noise as in Fig. 3b.

\section{PROPERTIES OF SPONGY-PARTICLE SUSPENSIONS}

At $t>\tau_{\mathrm{t}}$, properties of suspensions of spongy particles are studied as a function of the system density, for simulations performed with different realizations of the noise. Their behavior is compared to the limiting case of soft elastic particles with zero permeability by setting $\zeta^{*}=0$.

\section{A. Energy and stresses}

Figure 5 shows the different energy components per particle at $t>\tau_{\mathrm{t}}$ for systems with different dimensionless parameter $\zeta^{*}$ and as a function of the volume fraction $\varphi_{\text {free }}$. The

behavior of these systems with respect to the system density can be divided into three regimes. 


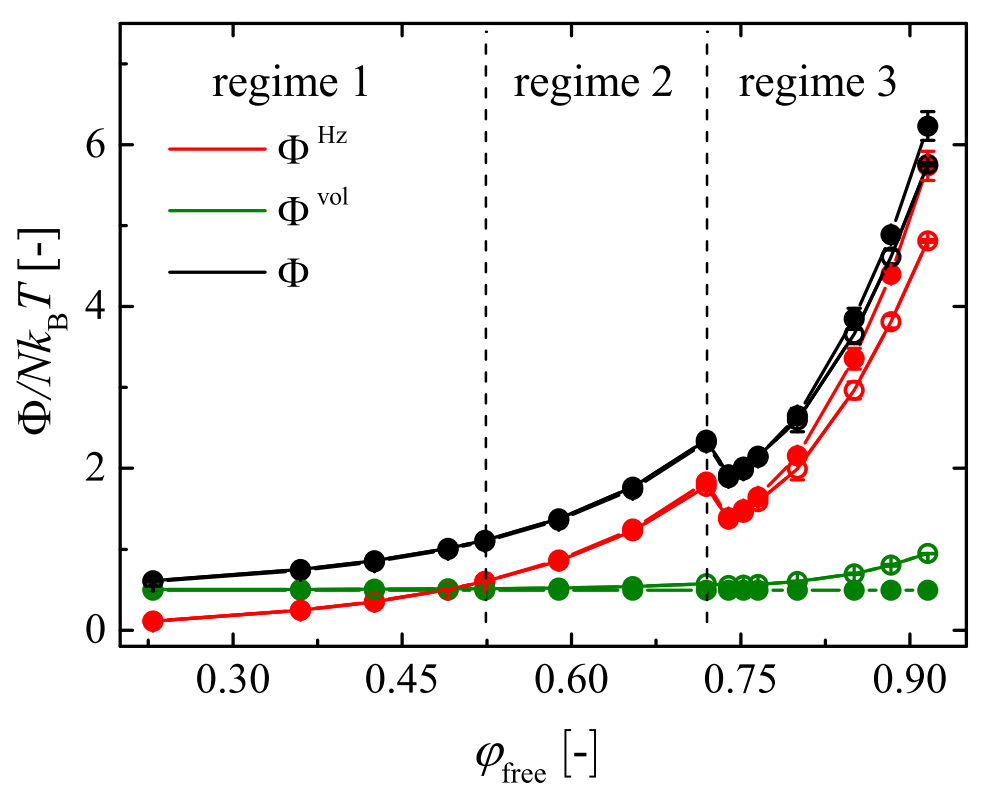

FIG. 5: Components of the energy per particle for $\zeta^{*}=0$ (closed symbols) and $\zeta^{*}=0.005$ (open symbols), and $S^{*}=2.5 \cdot 10^{-3}$. The error bars are based on the standard deviation obtained from running the simulation with 5 different realizations of the noise.

a. Regime 1 - This regime covers the following number densities and volume fraction ranges

$$
n \leq 0.8 \cdot 10^{19} \mathrm{~m}^{-3} \text { and } \varphi_{\text {free }} \leq 0.524 .
$$

At low system densities, the energy per particle is less than the thermal energy $k_{\mathrm{B}} T$. Particles do not overlap and the total interaction potential, Eq. (9), can be approximated by

$$
\Phi_{i}(\boldsymbol{\xi})=\Phi_{i}^{\mathrm{vol}}\left(R_{i}\right) .
$$

At equilibrium, the energy $\Phi_{i}^{\mathrm{vol}}$ and the driving force $-\partial_{R} \Phi_{i}^{\mathrm{vol}}$ vanish up to noise contributions. The particle-size fluctuations around $R_{\text {eq }}$ result in an average contribution to the energy of $k_{\mathrm{B}} T / 2$.

b. Regime 2 - In this regime, the number density and the corresponding volume fraction ranges are

$$
0.8 \cdot 10^{19}<n \leq 1.1 \cdot 10^{19} \mathrm{~m}^{-3} \text { and } 0.524<\varphi_{\text {free }} \leq 0.72 .
$$

While the volumetric energy remains independent of density as in the first regime, the contact between particles increases with increasing the particle volume fraction. In other 
words, particles deform due to contact but at no significant volume change. In this regime, the architecture of the individual particle, i.e. permeability, does not affect the overall behavior of the suspension.

c. Regime 3 - As the higher number densities and correspondingly volume fractions increase further,

$$
n>1.1 \cdot 10^{19} \mathrm{~m}^{-3} \text { and } \varphi_{\text {free }}>0.72,
$$

a transition in behavior occurs. It is pointed out that volume fractions well above unity, $\varphi_{\text {free }}>1$, can be reached at the expense of the particle compression. Regime 3 is characterized by an initial drop in the energy. The energy drop does not depend on the structure, i.e. the permeability, of the particles as it occurs for both porous and non-porous systems. It is to be noted that the system at a volume fraction of $\varphi_{\text {free }}=0.72\left(n=1.1 \cdot 10^{19} \mathrm{~m}^{-3}\right)$ does not show a transition to an ordered state even for $\tau_{\text {sim }}=3072 \tau_{Q}^{\mathrm{Br}}$ compared to Eq. (24). Consequently, the density at which energy drop occurs does not depend on the simulation time.

As the density of the system increases, the contact forces increase and particles reorganize into an ordered structure (see Fig. 2). Upon further increasing the number density, the volumetric energy deviates from the equilibrium value $\left(k_{\mathrm{B}} T / 2\right)$ to reduce contact forces between particles. Particle structure, i.e the permeability and respectively $\zeta^{*}$, affect the energy at number densities greater than at a volume fraction of $\varphi_{\text {free }}=0.78(n=1.2$. $10^{19} \mathrm{~m}^{-3}$ ), at which size-change becomes significant. This agrees with the work of Romeo et al. [9] where they studied the de-swelling behavior of microgels experimentally and they found that significant de-swelling occurs at volume fractions higher than 0.8.

As discussed before, the main difference between poroelastic and elastic particles is the ability to change size depending on the rate of deformation. Elastic particles can be elastically deformed upon Hertzian contact. Poroelastic particles, on the other hand, have an additional degree of freedom, that is their size. The volume deformation involved is rate-dependent as it depends on the permeating fluid as well as the structure of the particle expressed in terms of the permeability. This picture helps understanding the behavior shown in Fig. 5. This figure shows that at high system densities, porous particles change their size to reduce the Hertzian contact energy. Because elastic particles cannot accommodate volume changes, the resulting Hertzian contact energy is higher than in the case of porous 
particles.

It is to be noted that both contributions to the energy, the Hertzian contact energy and the volumetric contribution, share an explicit linear dependence on the particle elastic modulus (see Eq. (4) and Eq. (7)), while there is an additional implicit dependence on the elastic modulus in terms of the attained microstructures. With respect to increasing volume fraction $\varphi_{\text {free}}$, our results show that the increase in the energy contribution due to volume change is smaller than the increase in the energy contribution due to Hertzian contact (see Fig. 5), which is considered a non-trivial result. The contribution of these two components of energy result in a total energy that is lower for porous particles than elastic impermeable particles.

The effect of permeability can be further explained by studying the particle-size distribution at a certain time. Figure 6 shows histograms of particle radii for some of the systems shown in Fig. 5 at a volume fraction of $\varphi_{\text {free }}=0.916\left(n=1.4 \cdot 10^{19} \mathrm{~m}^{-3}\right)$. It is obvious from Fig. 6 that the mean of this distribution, $\langle R\rangle$, is lower than $R_{\text {eq }}$ for porous particles. The average size change observed for porous particles is small, approximately $1 \%$ of $R_{\text {eq }}$. In contrast, the particle size for the case of vanishing permeability remains at the initial distribution around $R_{\text {eq }}$. Both distributions result in non-vanishing volumetric contributions to the energy, $\Phi^{\mathrm{vol}}$. However, in the case of impermeable particles, the particle size can not change because of a vanishing transport coefficient, and therefore the particles can not adapt in order to attain a more favorable configuration. In other words, this means that a smaller fraction of phase space is accessible to the non-porous particles as compared to the porous particles. This implies that the relaxation to low-energy states of non-porous particle systems is less effective, as shown in Fig. 5.

The scenario of vanishing permeability can be translated to practical situations as follows. While vanishing permeability implies the absence of size relaxation, a small (but finite) permeability represents a system with a long (but finite) size relaxation. In this sense, the case of vanishing permeability studied above is representative of a system that has no noticeable size relaxation on the time-frame of the numerical simulations. Looking at the simulation results in Fig. 5 in this way, one can state that the system with vanishing permeability is not equilibrated, and even the system with the finite permeability may not be completely equilibrated yet. For this reason, these structures will be referred to as outof-equilibrium in the sequel. 

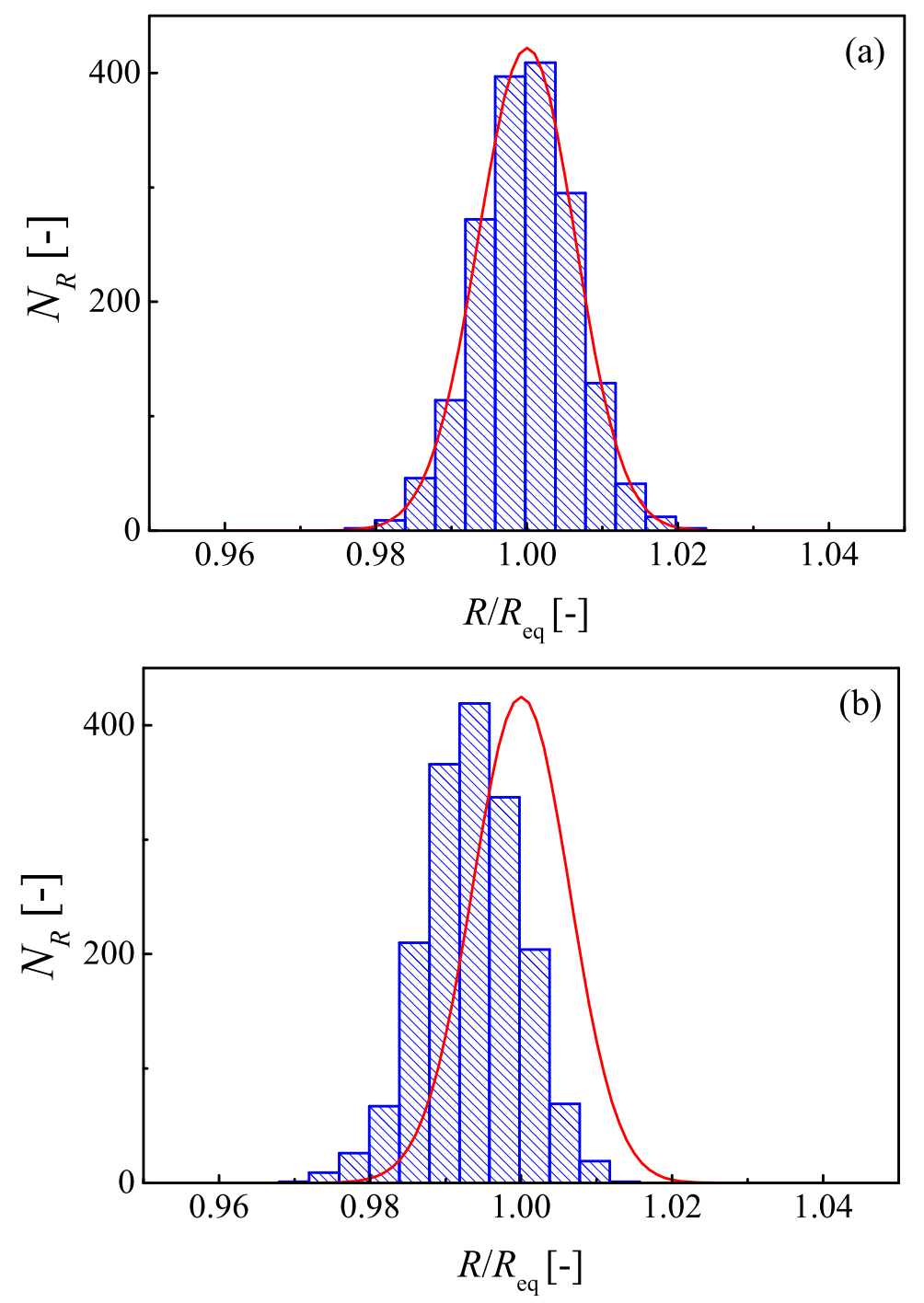

FIG. 6: Radius distribution at $t / \tau_{Q}^{\mathrm{Br}}=384.6$ for a system at $\varphi_{\text {free }}=0.916$ $\left(n=1.4 \cdot 10^{19} \mathrm{~m}^{-3}\right)$ with (a) $\zeta^{*}=0$ with $\langle R\rangle / R_{\text {eq }}=1$ and $\sigma_{R} / R_{\text {eq }}=0.00637$, and (b) $\zeta^{*}=0.005$ with $\langle R\rangle / R_{\text {eq }}=0.9934$ and $\sigma_{R} / R_{\text {eq }}=0.00643$. The red line represents the Boltzmann distribution for non-overlapping particles as given in Eq. (25).

The size changes in Fig. 6 are approximately $1 \%$ of $R_{\mathrm{eq}}$, which raises questions concerning the relevance of the volume contribution relative to the Hertzian contribution. The relative importance of both contributions can be compared most easily where they appear simultaneously in the evolution equations. This is the case for the first term on the righthand side of the size-dynamics equation Eq. (22b). Since both the Hertzian potential and the volumetric energy depend on the particle size, the driving force for size change can be expressed as $\tilde{F}_{R}=\tilde{F}_{R}^{\mathrm{Hz}}+\tilde{F}_{R}^{\mathrm{vol}}$, where $\tilde{F}_{R}^{\mathrm{Hz}}=-\partial_{\tilde{R}} \tilde{\Phi}^{\mathrm{Hz}}$ and $\tilde{F}_{R}^{\mathrm{vol}}=-\partial_{\tilde{R}} \tilde{\Phi}^{\text {vol }}$. The ratio of 
these contributions indicates their relative importance,

$$
\rho=\frac{\left|\left\langle\tilde{F}_{R}^{\mathrm{vol}}\right\rangle\right|}{\left|\left\langle\tilde{F}_{R}^{\mathrm{Hz}}\right\rangle\right|}
$$

where $\langle$.$\rangle denotes the average with respect to the statistical distribution from the simulation.$ For the specific system shown in Fig. 6 at $\varphi_{\text {free }}=0.916$, one finds $\rho \approx 17$, which shows quantitatively that the volumetric contribution is dominant in this case. It is pointed out that, for the system at hand, there is a significant difference between evaluating the forces using the average sizes and distances, versus evaluating the forces using the full statistical distribution; this holds not only for the forces, but also for the potential energy contributions.

The stresses are calculated using the constitutive relation [17]. Using the nondimensional interaction potential and scaling the length scales involved by $R_{\text {eq }}$, the stress tensor is given by

$$
\tilde{\boldsymbol{\sigma}}=\frac{\boldsymbol{\sigma}}{E}=n R_{\mathrm{eq}}^{3}\left[\left\langle\sum_{i}\left(\nabla_{\tilde{\boldsymbol{Q}}_{i}} \tilde{\Phi}\right) \tilde{\boldsymbol{Q}}_{i}\right\rangle+\alpha\left\langle\left(\partial_{\tilde{R}_{i}} \tilde{\Phi}\right) \tilde{R}_{i}\right\rangle \boldsymbol{I}\right],
$$

up to a constant pressure which takes into account the solvent pressure. The reader may refer to [17] for more information. The prefactor $\alpha$ indicates how much a particle follows the deformation of the containing volume. To put it more simply, one can think of this problem as a purely elastic problem in which a small volume change is applied to a volume that contains a spherical particle. If the particle is incompressible, the particle radius does not change and $\alpha=0$. On the contrary, if the particle is made of the same material as the surrounding material, $\alpha=1 / 3$, akin to affinely deforming the particle. In intermediate cases, it can be shown that $\alpha$ depends on the bulk modulus of the particle $K^{\text {part }}$ and the surrounding material $K^{\mathrm{sol}}$ as

$$
\alpha=\frac{K^{\mathrm{sol}}}{3 K^{\mathrm{part}}}
$$

Incompressible particles have an infinitely large bulk modulus $K^{\text {part }} \rightarrow \infty$ and $\alpha=0$, this is the value used for non-porous particles. For highly porous particles used in this study, one can assume that they are formed mostly of the surrounding solvent, so that their bulk moduli are comparable. For this reason, we use $\alpha=1 / 3$ for porous particles.

Figure 7 shows the average normal stress for non-porous $\left(\zeta^{*}=0\right)$ and porous $\left(\zeta^{*}=0.005\right)$ systems with $S^{*}=2.5 \cdot 10^{-3}$. The stress-density curve reflects the same features shown in Fig. 5 for the energy per particle. One can clearly see that the stress increases as the 
system density increases for both systems. However, in regime 3, this increase is stronger for non-porous particles. The reason for this is that, as explained before, in this regime the particle size change is large which reduces contact between particles and hence the stress is lower.

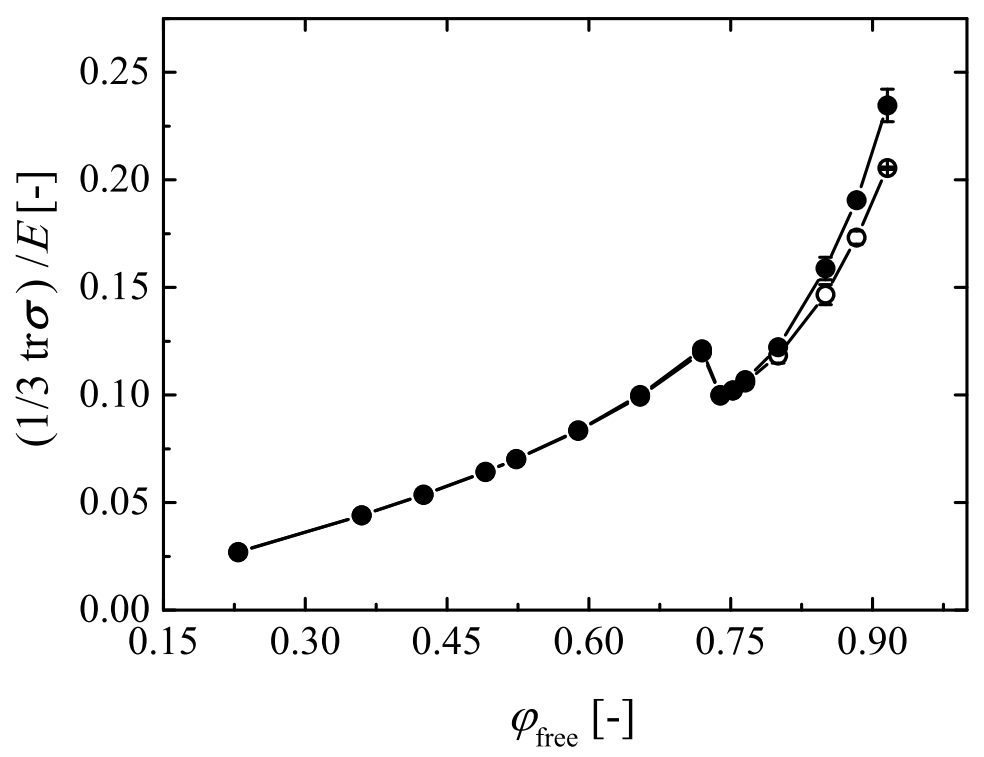

FIG. 7: Normal stress for systems with $\zeta^{*}=0$ (closed symbols) and $\zeta^{*}=0.005$ (open symbols), and $S^{*}=2.5 \cdot 10^{-3}$. The error bars are based on the standard deviation obtained from running the simulation with 5 different realizations of the noise.

\section{B. Diffusion}

The mobility of particles can be described by means of the diffusion tensor. A single submicron-sized particle in a solvent is subject to Brownian forces, and moves with a diffusion coefficient $D_{0}$ given by

$$
D_{0}=\frac{k_{\mathrm{B}} T}{6 \pi \eta R_{\mathrm{eq}}},
$$

which is equal to $8.58 \cdot 10^{-13} \mathrm{~m}^{2} / \mathrm{s}$ for the particles used in this study. The Mean Square Displacement (MSD) of this particle is defined as

$$
\left\langle\Delta \boldsymbol{r}(t)^{2}\right\rangle=\left\langle\left(\boldsymbol{r}(t)-\boldsymbol{r}\left(t_{0}\right)\right)^{2}\right\rangle .
$$

For a single-particle system, the mean square displacement is a linear function in time with a slope that is twice $D_{0}$. As more particles are added, their motion is hindered over longer 
times and the MSD- $t$ relation becomes non-linear. For a many-particle system, $\langle\cdot\rangle$ is the average over all particles and all samples extracted from the same simulation. A sample is defined based on the longest time scale for particle displacement which is, in this case, the Brownian time scale (see Table II). A sample is defined to run from an initial time $t_{0}$ for a period of $10 \tau_{Q}^{\mathrm{Br}}$. In general, if no deformation is applied, the MSD for a many particle system is given by

$$
\langle\Delta \boldsymbol{r}(t) \Delta \boldsymbol{r}(t)\rangle=2 \boldsymbol{D} t
$$

where $\boldsymbol{D}$ is the diffusion tensor. The off-diagonal components are zeros because the Brownian forces in different directions are uncorrelated. For a single particle system, the diagonal components of the diffusion tensor are $D_{x x}=D_{y y}=D_{z z}=D_{0}$. At short times, the slope of the MSD- $t$ curve gives the the short-time diffusion coefficient $D^{\mathrm{S}}$, which is equivalent to $D_{0}$ in the dilute limit. At longer times, the particles interact and their motion is hindered, and their diffusion coefficient is lowered. The long-time diffusion coefficient is denoted by $D^{\mathrm{L}}$.

Figure 8 shows the short-time diffusion coefficient for both systems shown in Fig. 5. It is calculated from the initial slope of the MSD curve. In regime 1, the volume fractions are low enough that particles initially diffuse with a coefficient that is, approximately, equal as $D_{0}$. This coefficient decreases as the particle volume fraction increases, in regime 2 and regime 3. In regime 3, porous particles can diffuse more than impermeable particles because of their ability to change their size which gives them, locally, more space.

Figure 9 shows that the long-time diffusion of these systems is lower than the short-time diffusion, as particles encounter other particles in the system. The decrease in long-time diffusion coefficient with increasing the particle volume fraction is steeper than the decrease in short-time diffusion coefficient. This steep decrease in $D^{\mathrm{L}}$ is attributed to the fact that particles need to travel shorter distances to encounter neighboring particles as the system density increases and that the caging becomes more effective. However, porous and nonporous particle systems show the same diffusive behavior over long times. This proves that the long-time diffusion is not sensitive to size change, but is dominated mainly by the density of the system. Based on the long-time diffusion coefficient, ordering occurs at $D^{\mathrm{L}} / D_{0} \approx 0.1$, which gives a dynamic criterion to the onset of regime 3 . This criterion $\left(D^{\mathrm{L}} / D_{0} \approx 0.1\right)$ was reported by Löwen and coworkers $[42,43]$ as a universal value for freezing of colloidal liquids into crystals for Brownian systems which was also confirmed using simulations for particles 


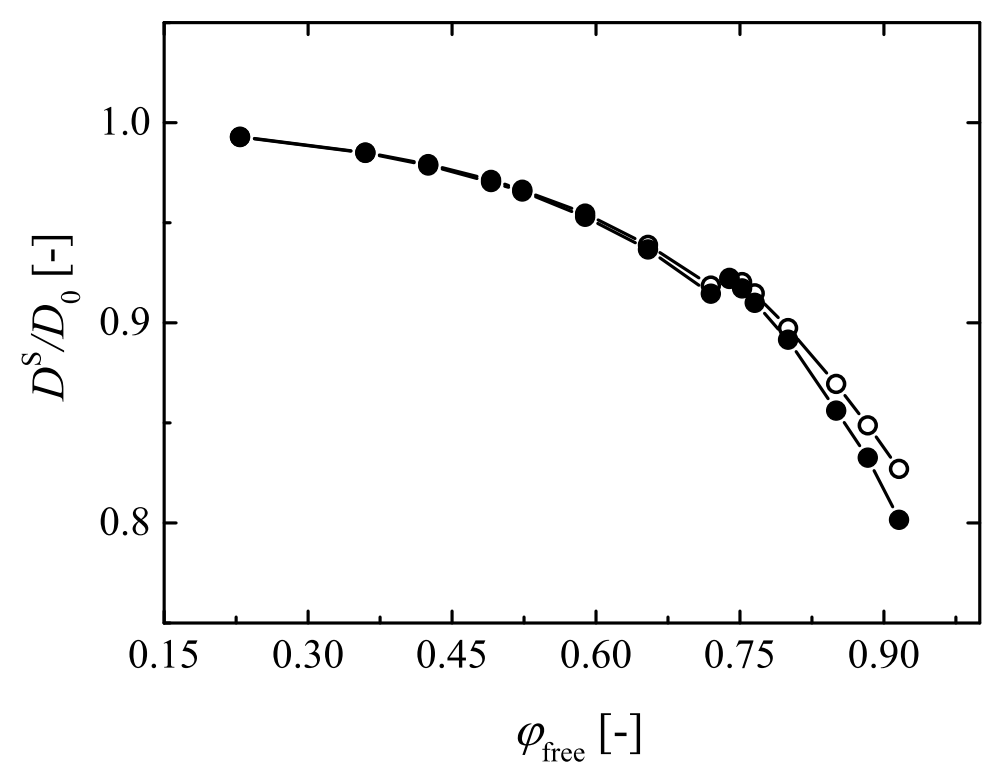

FIG. 8: Scaled short-time diffusion coefficient $D^{\mathrm{S}}$ for porous systems with $\zeta^{*}=0.005$ (open symbols) and non-porous systems with $\zeta^{*}=0$ (closed symbols), and $S^{*}=2.5 \cdot 10^{-3}$ at different number densities. $D_{0}$ denotes the single particle diffusion coefficient in the dilute limit calculated based on the equilibrium size. The standard error of the data based on simulations ran with 5 different realizations of the noise is smaller than the symbol size.

under Yukawa potential.

\section{STRUCTURE IN REGIME 3}

Regime 3, as discussed before, is the regime in which particles form ordered structures in order to reduce the total energy of the system. The transition to an ordered state depends on the system density (see Fig. 5). The interplay between packing, elastic properties, and permeability is a significant factor in the formation of crystalline colloidal systems. In this section, the effect of permeability on the transition to ordered structures is studied in detail in terms of the structure obtained as well as the structure evolution for highly dense systems. 


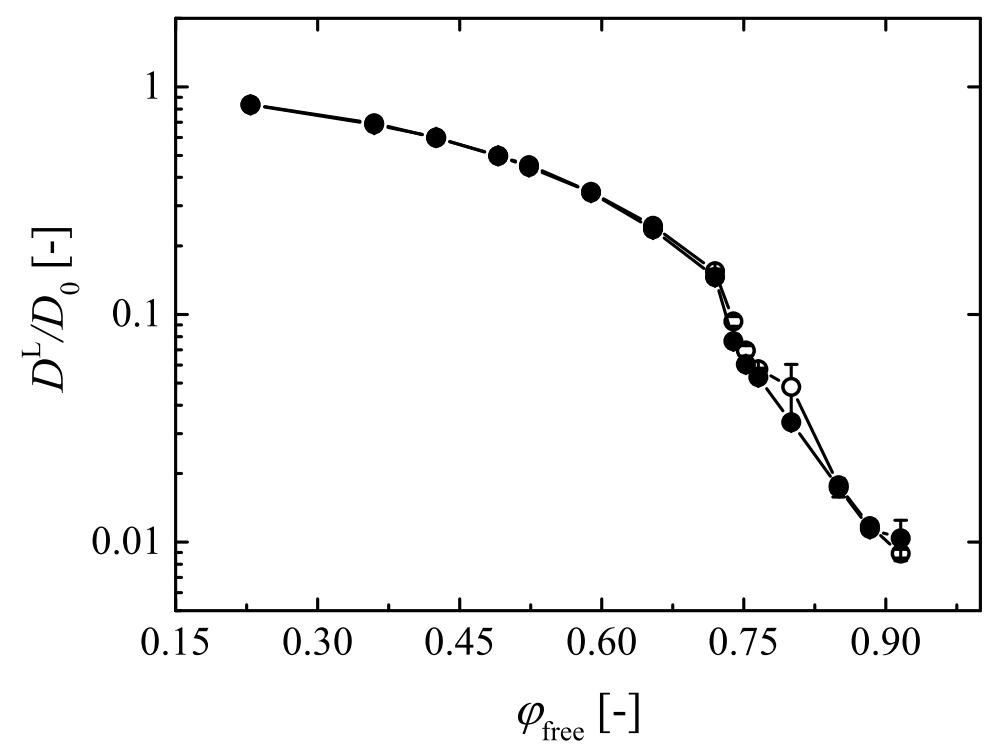

FIG. 9: Scaled long-time diffusion coefficient $D^{\mathrm{L}}$ for porous systems with $\zeta^{*}=0.005$ (open symbols) and non-porous systems with $\zeta^{*}=0$ (closed symbols), and $S^{*}=2.5 \cdot 10^{-3}$ at different number densities. $D_{0}$ denotes the single particle diffusion coefficient in the dilute limit calculated based on the equilibrium size. The error bars are the standard error of simulations ran with 5 different realizations of the noise.

\section{A. Average local-order parameter}

The structure is characterized by means of the average local-order parameter [44, 45] defined, for a particle $i$ with a number of neighboring particle $N_{b}$, as

$$
\bar{q}_{l}(i)=\sqrt{\frac{4 \pi}{2 l+1} \sum_{m=-l}^{l}\left|\bar{q}_{l m}(i)\right|^{2}},
$$

where

$$
\bar{q}_{l m}(i)=\frac{q_{l m}(i)+\sum_{k=1}^{N_{b}} q_{l m}(k)}{N_{b}+1},
$$

and

$$
q_{l m}(i)=\frac{\sum_{j=1}^{N_{b}} Y_{l m}\left(\boldsymbol{r}_{i j}\right)}{N_{b}} .
$$

In the above equations, $q_{l m}$ is a complex number that depends on all the spherical harmonics $Y_{l m}$ of order $l$ with integers $m \in\{-l, \ldots, l\}$ for a pair of particles with interparticle vector $\boldsymbol{r}_{i j}$ measured relative to a fixed frame of reference. Particles are defined as neighbors when the 
interparticle separation corresponds to the first minimum in the radial distribution curve. In Eq. (35), $q_{l m}$ is averaged over the neighbors of particle $i$ in addition to particle $i$ itself, which increases the accuracy in discriminating different crystal structures [44].

The order of the spherical harmonics used are $l=4$ and $l=6$ to capture structures with four-fold symmetry like the BCC, and structure with six-fold symmetry like HCP, respectively. Particles are considered to be solid-like if $\bar{q}_{6} \geq 0.29$ and they are further categorized as BCC for $\bar{q}_{4} \leq 0.05, \mathrm{HCP}$ for $0.05<\bar{q}_{4} \leq 0.1$, and FCC for $\bar{q}_{4}>0.1[44,46]$. Using the average local-order parameter, one can express the structure in terms of fractions of BCC, HCP and FCC crystals as well as amorphous phase.

\section{B. Final structure}

Figure 10 shows the average composition $f$ of the different crystals for systems with $\zeta^{*}=0$ and $\zeta^{*}=0.005$ at different system densities. The BCC is not favored for both systems because is has only four-fold symmetry and is unstable; even if it develops at earlier time during a simulation, it soon changes into the more stable FCC or HCP. The structure obtained, in both cases and for the entire range of number densities shown, is the random hexagonal close packing (rHCP) which is a mixture of HCP and FCC. HCP and FCC have energies that are a fraction of $k_{\mathrm{B}} T$ different in favor of FCC [36, 47].

Figure 10 characterizes the structure in the low-energy states, that is at times longer than $\tau_{\mathrm{t}}$. The fact that the error bars are relatively large points at a significant influence of the different realizations of the noise, i.e. of the history. It appears that, within standard errors, no clear trends can be seen in the density-dependence of the structure obtained at times longer than $\tau_{\mathrm{t}}$, and a systematic difference between porous and non-porous systems is not evident. This is to be compared with the energy results presented in Fig. 5, which did show a dependence on the permeability. While the energy is a useful (single) physical quantity that captures some features of the structure, it is more illuminating to examine the various crystalline phases present in the system, and how these phases actually emerge, which is discussed in the following section. 


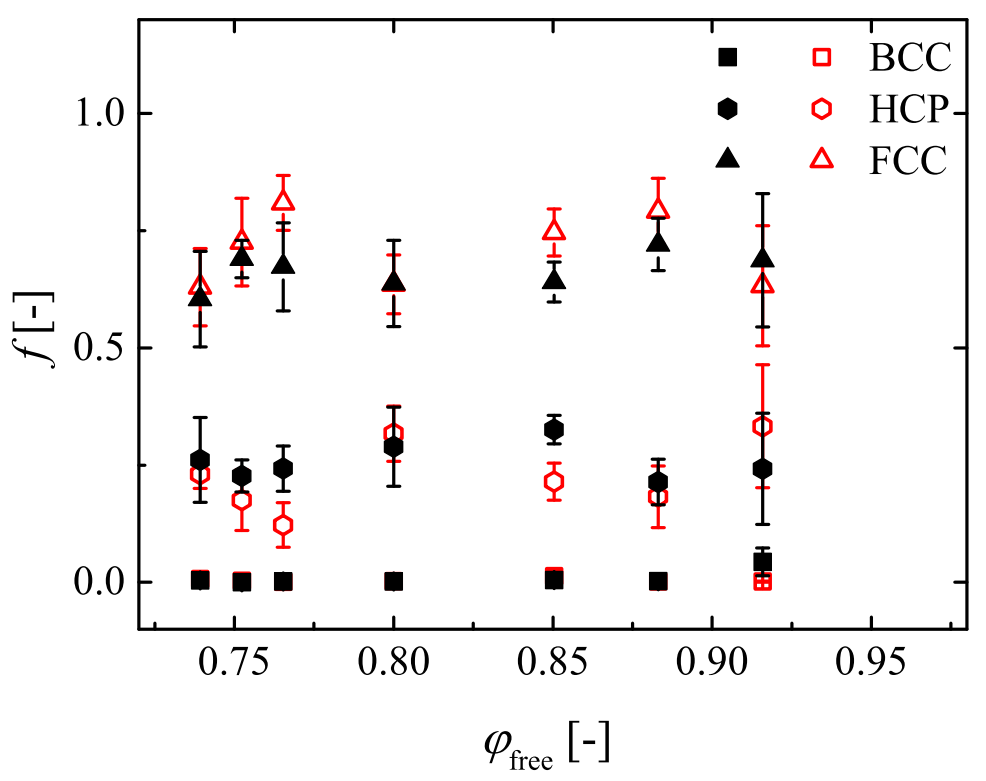

FIG. 10: Average structural composition $f$ of BCC, HCP, and FCC structures for systems with $S^{*}=2.5 \cdot 10^{-3}$ and $\zeta^{*}=0.0$ (closed symbols) and $\zeta^{*}=0.005$ (open symbols). Error bars are the standard error based on simulations run at 5 different realizations of the noise.

\section{Structure evolution}

The evolution of structure of both systems, shown in Fig. 5, are studied in this section. As mentioned before, the average final structure of the suspensions studied depends on the realization of the noise used in a simulation. To that end, in this section, we compare two typical systems with $\zeta^{*}=0$ and $\zeta^{*}=0.005$ at $\varphi_{\text {free }}=0.916\left(n=1.4 \cdot 10^{19} \mathrm{~m}^{-3}\right)$ and $S^{*}=2.5 \cdot 10^{-3}$ using a certain realization of the noise. The dynamics of structure formation is expressed in terms of the transition time.

Figure 11 shows the $\left(\bar{q}_{4}, \bar{q}_{6}\right)$-density maps as a function of time. The bottom row in Fig. 11 shows that at $2 \tau_{\mathrm{t}}$ most of the particles in both cases are solid-like, based on the definition given in Sec. VA, irrespective of their bias to one phase or the other (HCP/FCC). This is confirmed from Fig. 12 where the amount of crystalline structure, defined as $f^{\text {cryst }}=$ $f^{\mathrm{BCC}}+f^{\mathrm{HCP}}+f^{\mathrm{FCC}}$, for these systems is shown as a function of time. It becomes clear that at times greater than $\tau_{\mathrm{t}}$ both systems have fully crystallized.

The left column in Fig. 11 shows the result for elastic particles with zero permeability. At $t=0.5 \tau_{\mathrm{t}}$ (top row in Fig. 11), most of the particles are still in the amorphous state with 
some clusters forming HCP/FCC. At $t=\tau_{\mathrm{t}}$ (middle row in Fig. 11), more clusters with $\mathrm{HCP} / \mathrm{FCC}$ structure form, that grow over time. This result is qualitatively similar to results obtained for the Yukawa systems in [46] in which they studied the effect of the interaction energy, which can be related to $S^{*}$ in our model, on the crystallization dynamics. Here, we build on the knowledge about the crystallization process [46] by studying the effect of the additional degree of freedom, the particle radius. The right column in Fig. 11 shows the structure evolution for porous particles. Porous particles at $0.5 \tau_{\mathrm{t}}$ (top row in Fig. 11) show the same behavior as non-porous particles, that is most of the particles are in the amorphous phase. At $\tau_{\mathrm{t}}$ (middle row in Fig. 11), porous particles are mostly in the FCC domain, while non-porous particles are distributed over the FCC, HCP and amorphous domains, respectively. This suggests that the formation of the stable FCC structure occurs faster for porous particles than for non-porous particles in terms of times relative to $\tau_{\mathrm{t}}$. To further investigate this effect, the number of clusters of ordered particles is studied as a function of time.

The number of clusters is calculated based on particles that have a BCC, HCP or FCC structure, in other words, particles with $\bar{q}_{6} \geq 0.29$. If the distance between a particle and its neighbor is smaller than a certain threshold, they belong to the same cluster. The threshold in this case is set to the first minimum in the radial distribution function calculated based on the position of particles with $\bar{q}_{6} \geq 0.29$. Figure 13 shows the evolution of the number of clusters over time for both systems shown in Fig. 11. Clusters can be seen as nuclei from which ordered structures grow. Figure 13 shows that, in time-units of $\tau_{\mathrm{t}}$, porous particles form slightly fewer clusters, and a slightly higher degree of crystallinity (see Fig. 12), than non-porous ones. This can be interpreted as the size-dynamics allowing more particles to locally re-arrange relative to their neighbors, which accelerates crystal growth into the stable structure, that is FCC for our system. This conclusion is supported by the fact that porous particles diffuse more than non-porous particles as indicated by the short time diffusion coefficient shown in Sec. IV B.

The cluster-size distribution for the same systems, that is with $\zeta^{*}=0$ and $\zeta^{*}=0.005$ at $\varphi_{\text {free }}=0.916\left(n=1.4 \cdot 10^{19} \mathrm{~m}^{-3}\right)$ and $S^{*}=2.5 \cdot 10^{-3}$ using 5 different realizations of the noise, is analyzed in terms of moments of the cluster-size distribution. Here, we use the definition of the number- and weight-average molecular weights, $M_{n}$ and $M_{w}$, respectively [48], with $M_{w} / M_{n}=1$ being representative of a mono-disperse and $M_{w} / M_{n} \gg 1$ of a strongly 

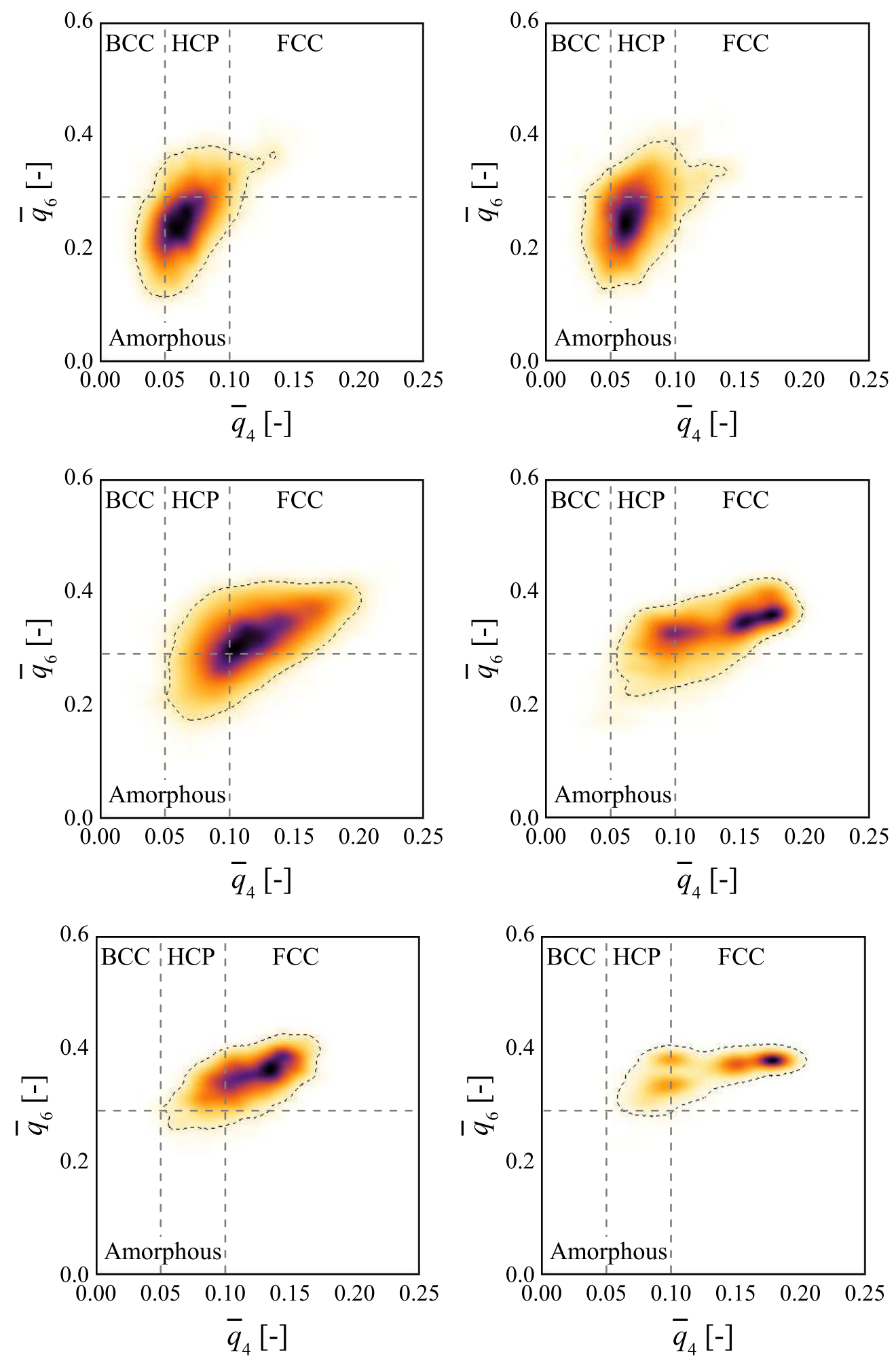

FIG. 11: Order parameter $\left(\bar{q}_{4}, \bar{q}_{6}\right)$-density maps at $t=0.5 \tau_{\mathrm{t}}$ (top row), at $t=\tau_{\mathrm{t}}$ (middle row) and at $t=2 \tau_{\mathrm{t}}$ (bottom row) for systems with $\varphi_{\text {free }}=0.916\left(n=1.4 \cdot 10^{19} \mathrm{~m}^{-3}\right)$ and $\zeta^{*}=0$ (left column), and $\zeta^{*}=0.005$ (right column), respectively. The contour lines correspond to $5 \%$ of the maximum density, for better visualization. 


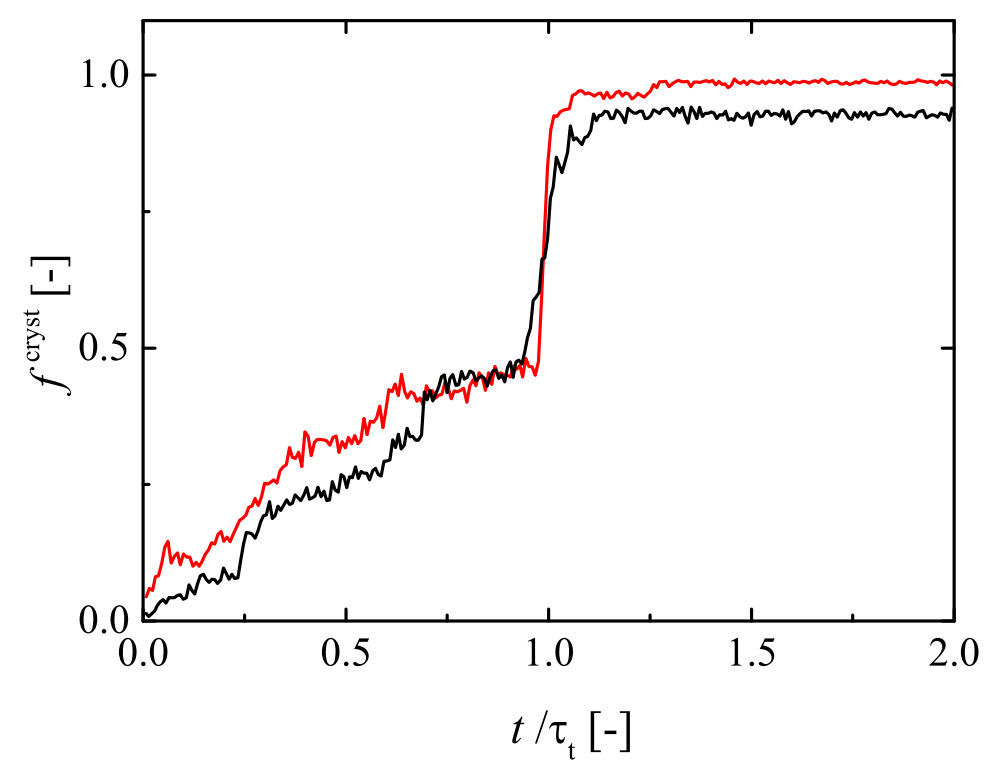

FIG. 12: Evolution of fraction of crystalline phase for the system in Fig. 11 at a density of $\varphi_{\text {free }}=0.916\left(n=1.4 \cdot 10^{19} \mathrm{~m}^{-3}\right)$ with $S^{*}=2.5 \cdot 10^{-3}$ and $\zeta^{*}=0$ (black), and $\zeta^{*}=0.005$ (red), respectively.

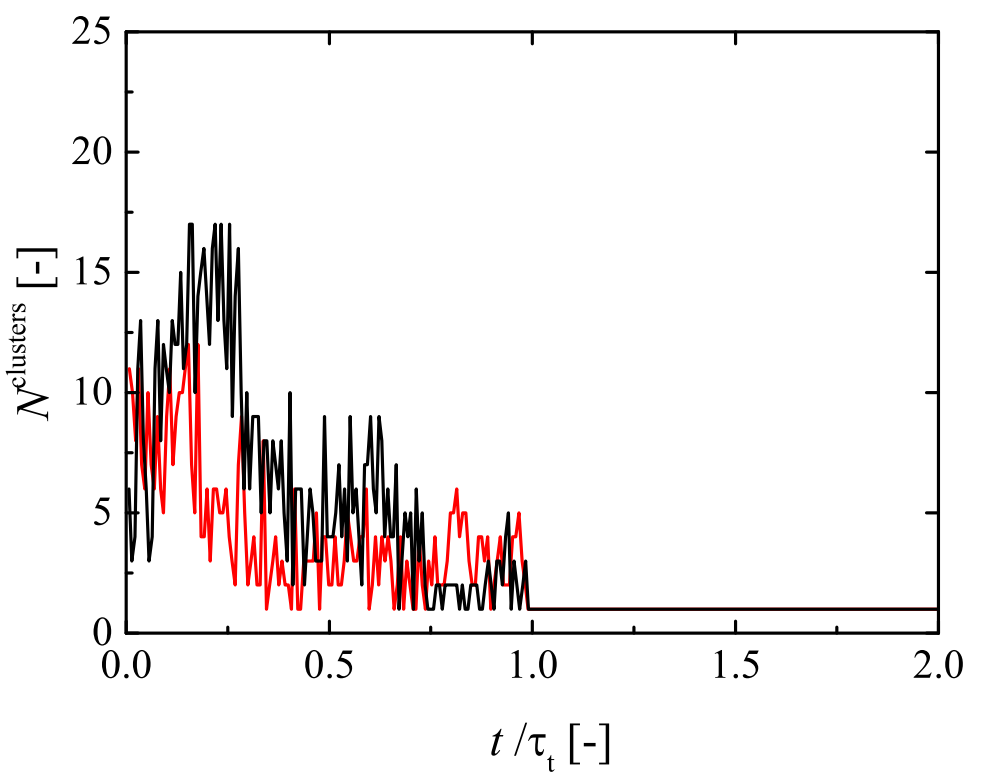

FIG. 13: Evolution of number of clusters of ordered particles for the system in Fig. 11 at a density of $\varphi_{\text {free }}=0.916\left(n=1.4 \cdot 10^{19} \mathrm{~m}^{-3}\right)$ with $S^{*}=2.5 \cdot 10^{-3}$ and $\zeta^{*}=0$ (black), and $\zeta^{*}=0.005$ (red), respectively. 
poly-disperse distribution. The cluster size is expressed in terms the number of particles forming this cluster. Figure 14 shows that, at $t<\tau_{\mathrm{t}}$, the average cluster size given by $M_{n}$ of porous particle systems is higher in comparison with non-porous particles. This confirms that porous particle systems grow larger clusters on shorter time scales than non-porous systems. This is attributed to the fact that porous particles are locally more mobile, which is established by the higher short-time diffusion in regime 3 (see Sec. IV B). On the other hand, the polydispersity in cluster-size distribution, i.e. $M_{w} / M_{n}$, is shown in Fig. 15. It can be seen that, on average, the polydipersity of non-porous particles is higher that the polydispersity of porous particles.

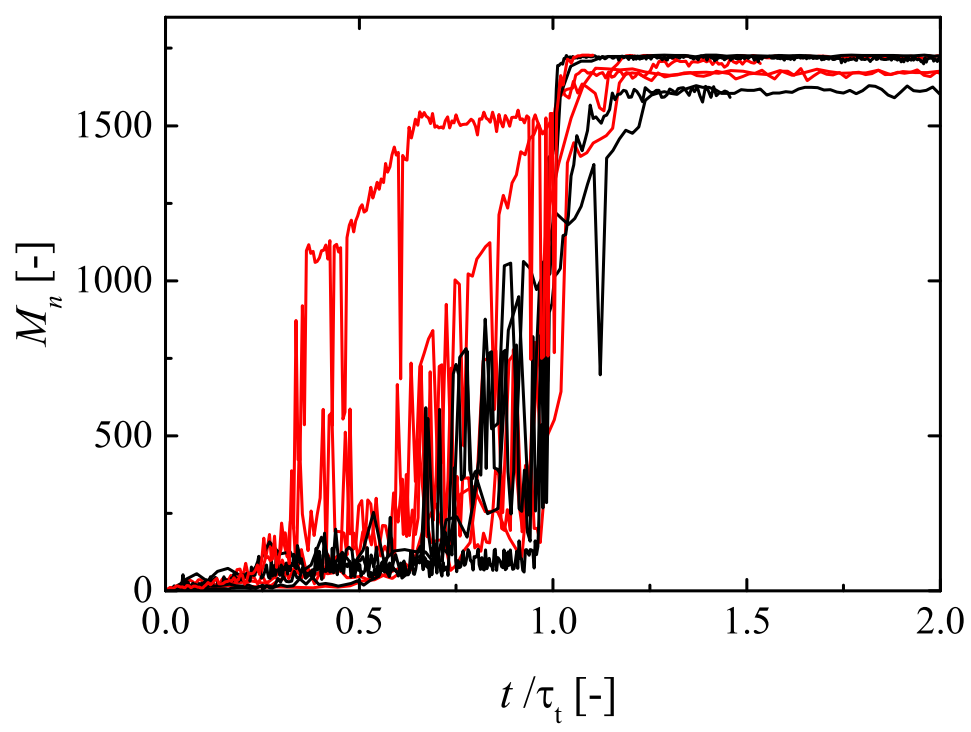

FIG. 14: The first moment of cluster-size distribution $M_{n}$ of ordered particles for systems with different realizations of the noise at a density of $\varphi_{\text {free }}=0.916\left(n=1.4 \cdot 10^{19} \mathrm{~m}^{-3}\right)$ with $S^{*}=2.5 \cdot 10^{-3}$ and $\zeta^{*}=0$ (black) and $\zeta^{*}=0.005$ (red).

\section{CONCLUSIONS}

In this paper, the rate-dependent behavior of spongy particles and its effect on the effective overall properties of suspensions made from such particles is studied in detail. The permeability of the particles and the viscosity of the permeating solvent are responsible for the rate-dependent size-change of these particles. This work applies the dynamic two-scale developed by Hütter et al. [17] to suspensions of spongy particles. The model accounts for 


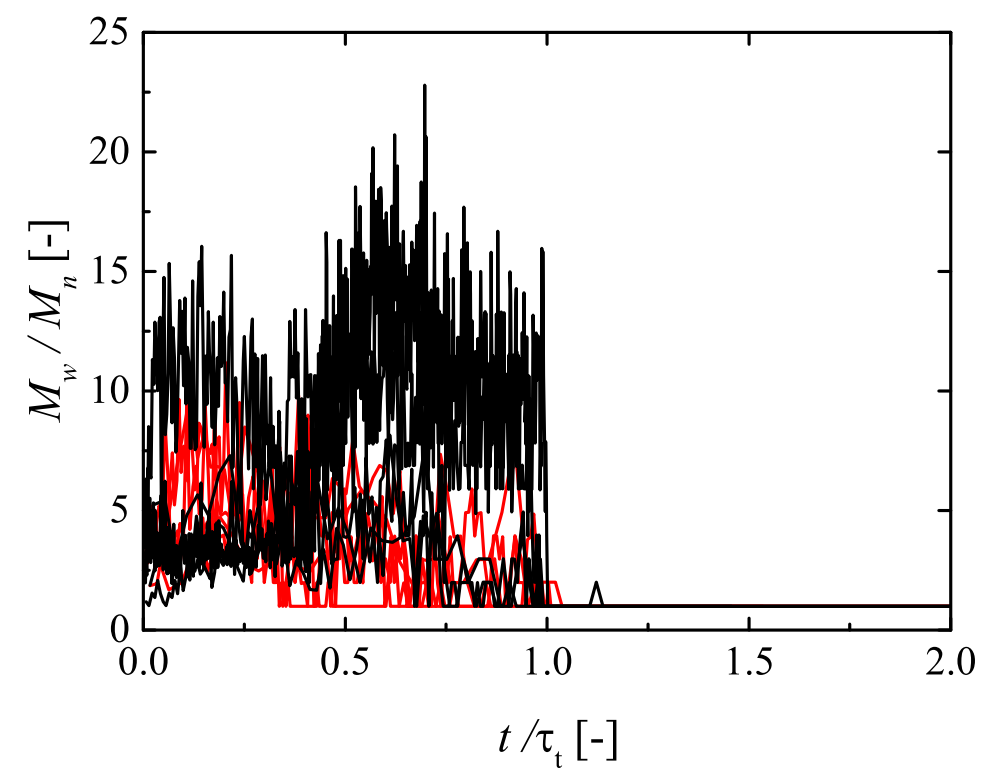

FIG. 15: The polydispersity in cluster-size distribution $M_{w} / M_{n}$ of ordered particles for systems with different realizations of the noise at a density of $\varphi_{\text {free }}=0.916$ $\left(n=1.4 \cdot 10^{19} \mathrm{~m}^{-3}\right)$ with $S^{*}=2.5 \cdot 10^{-3}$ and $\zeta^{*}=0$ (black) and $\zeta^{*}=0.005$ (red).

the rate-dependent size change of these particles by considering the particle size as a separate dynamic degree of freedom. The specific system realization is achieved by making a choice on the potential energy and the friction coefficients. Particles are assumed to interact through a purely repulsive potential due to, (i) the elastic shape change upon contact, and (ii) the volume change of particles compared to their size in the dilute limit. Particle-based numerical simulations are performed with the purpose of studying the effect of the ratedependent particle softness ascribed to the particle architecture, i.e. permeability, on the transient and long-time behavior of spongy-particle suspensions in the absence of applied deformation. Understanding the behavior in equilibrium, i.e. in the absence of applied deformation, is an essential prerequisite for understanding the behavior under deformation (e.g. shear).

At high densities, the suspension undergoes a fast transition from an initial high-energy state to a long-lived (glassy) state, which in turn evolves to an ordered low-energy state. The second transition to the ordered state occurs on time scales significantly larger than the Brownian time scale. By performing a careful study of numerical parameters, the transition time is found to depend significantly on the sample history while the final state is rather 
insensitive to the sample history, where the latter is changed by varying the initial state and the realization of the noise. At times larger than the transition time, the permeability has a significant effect on the overall response at packing fractions higher than 0.8. Porous particles at such high packing densities reduce their size in order to reduce the contact forces. Consequently, the energy and the normal stress are lower compared to impermeable particles. Porous particles are also found to be, locally, more mobile as observed by the higher short-time diffusion coefficient.

This work also addresses the crystallization phenomena for colloidal systems. Upon increasing the volume fraction of particles, the energy in the system increases gradually. At volume fractions around 0.72 the energy drops, which is found to coincide with the formation of crystals. Increasing the volume fraction even further, the energy of the crystalline structures gradually increases. Specifically at volume fraction of 0.72 , the long-time diffusion coefficient is one-tenth of the single particle diffusion coefficient, consistent with the universal value for the freezing point of colloidal liquids into crystals $[42,43]$. We have studied the structures formed and their evolution, in time units of the transition time $\tau_{\mathrm{t}}$, by means of the averaged bond-order parameter $[44,45]$. We show that, although the final structure is not qualitatively affected by the permeability of the particles, the growth of the stable FCC structure is accelerated for porous particle systems. In this case, bigger clusters are created faster, as opposed to their impermeable counterpart.

Previous models have captured part of the general features of soft-particle suspensions by considering only the elastic nature of softness. However, this work draws attention to taking into account the rate-dependent particle softness on the long-time behavior and dynamics of suspensions made from such particles, even at radius changes as small as $1 \%$. On the one hand, the elastic softness alone, if applied to spongy particles, overestimates the longtime values of energy and stress at volume fractions higher than 0.8 . This $80 \%$-limit is consistent with experimental observations that the compression of particles becomes more significant at these volume fractions [9]. It is pointed out that porous particles adjust their size in a dissipative way to reduce contact forces at high packing-densities, which cannot be captured by considering solely the (non-dissipative) elastic particle-softness. On the other hand, we have found that, even at radius changes as small as $1 \%$, the rate-dependent softness attributed to porous particles accelerates the dynamics of the system, as evidenced by the higher short-time diffusion coefficient. The effect of the accelerated dynamics is twofold. 
First, transitions to the ordered state occurs on shorter time scales, and second the growth of stable structures, which is FCC for our systems, is accelerated.

In future work, this model will be applied to systems under deformation in order to study the mechanical behavior of these suspensions. It has been noted in the literature (see for example [14] and references therein) that not accounting for internal degrees of freedom falls short of explaining, for instance, the increase in yield stress of soft-particle systems compared to hard-particle systems. We hypothesize that incorporating size dynamics would provide insight in the description of such phenomena, particularly under strong flow conditions.

\section{Appendix A: Hertzian potential}

This appendix is concerned with determining the coefficients in Eq. (5) for the Hertzian potential referred to in Sec. II A.

Consider two deformable particles $i$ and $j$ with radii $R_{i}$ and $R_{j}$ centered at $\boldsymbol{Q}_{i}$ and $\boldsymbol{Q}_{j}$ respectively. Particles are in contact with an amount of overlap of $h_{i j}=R_{i}+R_{j}-\left|\boldsymbol{Q}_{i j}\right|$ where $\left|\boldsymbol{Q}_{i j}\right|=\left|\boldsymbol{Q}_{i}-\boldsymbol{Q}_{j}\right|$. In this case, the energy associated with the elastic contact between these two particles is

$$
\Phi_{i j}^{\mathrm{Hz}}= \begin{cases}\frac{E}{2\left(1-\nu^{2}\right)} h_{i j}^{n} R_{c}^{3-n}+k & h_{i j}>0, \\ 0 & h_{i j} \leq 0 .\end{cases}
$$

For small deformations, constants $C, n$ and $K$ in Eq. (A1) follow from Hertz theory [23], $C=\frac{8}{15}, n=\frac{5}{2}$ and $k=0$. This is valid for $\frac{h_{i j}}{R_{i}+R_{j}}<0.1$. The values of these constants vary for larger deformations. Liu et al. [25] performed experiments on a single particle deformed between two flat plates. They found that the force is a function of indentations to the power 3 for values between $10 \%$ and $20 \%$ of the diameter of the particle and to the power 5 for values higher than 20\%. Based on the information known about the small deformation range, the remaining constants can be calculated to ensure force and energy continuity assuming that all the particles are at their equilibrium size, i.e. $R_{i}=R_{j}=R_{\text {eq. }}$. This gives the following $C, n$ and $k$ for different ranges of deformation

$$
\begin{cases}C=\frac{8}{15}, n=\frac{5}{2}, k=0 & h_{i j}^{*}<0.1, \\ C=\frac{5}{6} \sqrt{\frac{5}{2}}, n=4, k=\frac{\sqrt{10}}{1250} E^{*} R_{\mathrm{eq}}^{3} & 0.1 \leq h_{i j}^{*}<0.2, \\ C=\frac{125}{144} \sqrt{\frac{5}{2}}, n=6, k=\frac{89 \sqrt{10}}{11250} E^{*} R_{\mathrm{eq}}^{3} & 0.2 \leq h_{i j}^{*}<0.6,\end{cases}
$$


where $h_{i j}^{*}=\frac{h_{i j}}{R_{i}+R_{j}}$ and $E^{*}=\frac{E}{2\left(1-\nu^{2}\right)}$.

\section{Appendix B: Effect of system size}

In this appendix, the effect of the system size on the overall behavior is studied. This is done for the three regimes described in Sec. IV. Three systems, with numbers of particles $N_{1}=12^{3}, N_{2}=17^{3}$ and $N_{3}=22^{3}$, are studied. Particles are initially placed on a simplecubic lattice to ensure that the initial configuration is the same for all systems. For each system, averages are calculated from five simulations with different realizations of the noise

Table III shows that the effect of the system size on the energy per particle is negligible in the first two regimes, and moderate in the third regime. The situation is different for the transition time. It can be seen in Table IV that the transition time systematically decreases upon increasing the number of particles, at the same number density. This trend is as expected, in view of the periodic boundary conditions. For increasing system size, the influence of the boundary conditions on the system behavior becomes less. More precisely, the larger the system, the less does the system feel the constraints imposed by the boundary conditions. And, in turn, less constraints on the dynamics implies that the system manages to find more rapidly the path to a more favorable state.

TABLE III: Energy $\left(\Phi / N k_{\mathrm{B}} T\right)$ for a system with $N_{1}=12^{3}, N_{2}=17^{3}$ and $N_{3}=22^{3}$ particles, at different number densities. The standard deviation is based on five simulations each with different realizations of the noise.

\begin{tabular}{c|c|c|c}
$n \cdot 10^{19} \mathrm{~m}^{-3}$ & $N_{1}=12^{3}$ & $N_{2}=17^{3}$ & $N_{3}=22^{3}$ \\
\hline 1.0 & $1.7662 \pm 0.00045$ & $1.7691 \pm 0.00094$ & $1.7692 \pm 0.00035$ \\
1.223 & $2.5964 \pm 0.1430$ & $2.6172 \pm 0.03992$ & $2.7996 \pm 0.1729$ \\
1.350 & $4.6131 \pm 0.08706$ & $4.7178 \pm 0.1543$ & $5.1168 \pm 0.3852$
\end{tabular}


TABLE IV: Transition time for systems with $N_{1}=12^{3}, N_{2}=17^{3}$ and $N_{3}=22^{3}$ at different number density. The standard error is based on the different realizations of the noise.

\begin{tabular}{c|c|c|c}
$n \cdot 10^{19} \mathrm{~m}^{-3}$ & $N_{1}=12^{3}$ & $N_{2}=17^{3}$ & $N_{3}=22^{3}$ \\
\hline 1.0 & - & - & - \\
1.223 & $10.4172 \pm 5.1237 \mathrm{~s}$ & $4.5828 \pm 1.1284 \mathrm{~s}$ & $4.2550 \pm 1.6232 \mathrm{~s}$ \\
1.350 & $15.1270 \pm 3.9855 \mathrm{~s}$ & $11.6080 \pm 1.9049 \mathrm{~s}$ & $9.6067 \pm 3.1361 \mathrm{~s}$
\end{tabular}

\section{ACKNOWLEDGMENTS}

This work forms part of the research programme of the Dutch Polymer Institute (DPI), project \#738

[1] A. Bouchoux, P.-E. Cayemitte, J. Jardin, G. Gésan-Guiziou, and B. Cabane, Biophys. J. 96, 693 (2009).

[2] N. L. Thomas, Prog. Org. Coat. 19, 101 (1991).

[3] J. B. Thorne, G. J. Vine, and M. J. Snowden, Colloid Polym. Sci. 289, 625 (2011).

[4] C. W. MacMinn, E. R. Dufresne, and J. S. Wettlaufer, Phys. Rev. X 5, 011020 (2015).

[5] H. M. Wyss, T. Franke, E. Mele, and D. A. Weitz, Soft Matter 6, 4550 (2010).

[6] P. Snabre and P. Mills, Colloids Surf., A 152, 79 (1999).

[7] S. Adams, W. J. Frith, and J. R. Stokes, J. Rheol. 48, 1195 (2004).

[8] D. Vlassopoulos and M. Cloitre, Curr. Opin. Colloid Interface Sci. 19, 561 (2014).

[9] G. Romeo, L. Imperiali, J.-W. Kim, A. Fernández-Nieves, and D. Weitz, J. Chem. Phys. 136, 124905 (2012).

[10] S. V. Franklin and M. D. Shattuck, eds., Handbook of Granular Materials (CRC Press, 2015).

[11] J. Bouchard, J. Phys. I France 2, 1705 (1992).

[12] P. Sollich, F. Lequeux, P. Hébraud, and M. E. Cates, Phys. Rev. Lett. 78, 2020 (1997).

[13] P. Sollich, Phys. Rev. E 58, 738 (1998).

[14] A. Ikeda, L. Berthier, and P. Sollich, Soft Matter 9, 7669 (2013).

[15] M. Van Hecke, J. Phys.: Condens. Matter 22, 033101 (2009).

[16] J. R. Seth, L. Mohan, C. Locatelli-Champagne, M. Cloitre, and R. T. Bonnecaze, Nat Mater 
10, $838(2011)$.

[17] M. Hütter, T. J. Faber, and H. M. Wyss, Farad. Discuss. 158, 407 (2012).

[18] M. Grmela and H. C. Öttinger, Phys. Rev. E 56, 6620 (1997).

[19] H. C. Öttinger and M. Grmela, Phys. Rev. E 56, 6633 (1997).

[20] H. C. Öttinger, Beyond Equilibrium Thermodynamics (John Wiley \& Sons, 2005).

[21] A. Fernandez-Nieves and A. M. Puertas, Fluids, Colloids and Soft Materials: An Introduction to Soft Matter Physics, Vol. 7 (Wiley Online Library, 2016).

[22] C. L. A. Berli and D. Quemada, Langmuir 16, 7968 (2000).

[23] K. L. Johnson, Contact Mechanics (Cambridge University Press, 1985).

[24] J. R. Seth, M. Cloitre, and R. T. Bonnecaze, J. Rheol. 50, 353 (2006).

[25] K. K. Liu, D. R. Williams, and B. J. Briscoe, J. Phys. D: Appl. Phys. 31, 294 (1998).

[26] F. Beer, E. Johnston, and J. DeWolf, Mechanics of Materials (2002).

[27] H. C. Öttinger, Stochastic Processes in Polymeric Fluids: Tools and Examples for Developing Simulation Algorithms (Springer, 2012).

[28] G. K. Batchelor, An Introduction to Fluid Dynamics (Cambridge University Press, 2000).

[29] A. Verruijt, Introduction to Soil Dynamics (Springer, 2010).

[30] A. W. Lees and S. F. Edwards, J. Phys. C: Solid State Phys. 5, 1921 (1972).

[31] A. Quarteroni, R. Sacco, and F. Saleri, Numerical Mathematics, Texts in Applied Mathematics (Springer Berlin Heidelberg, 2010).

[32] C. A. Grattoni, H. H. Al-Sharji, C. Yang, A. H. Muggeridge, and R. W. Zimmerman, J. Colloid Interface Sci. 240, 601 (2001).

[33] A. Banerjee, M. Arha, S. Choudhary, R. S. Ashton, S. R. Bhatia, D. V. Schaffer, and R. S. Kane, Biomaterials 30, 4695 (2009).

[34] J. J. Liétor-Santos, B. Sierra-Martín, and A. Fernández-Nieves, Phys. Rev. E 84, 060402 (2011).

[35] P. H. J. Kouwer, M. Koepf, V. A. A. Le Sage, M. Jaspers, A. M. van Buul, Z. H. EksteenAkeroyd, T. Woltinge, E. Schwartz, H. J. Kitto, R. Hoogenboom, S. J. Picken, R. J. M. Nolte, E. Mendes, and A. E. Rowan, Nature 493, 651 (2013).

[36] A. P. Gast and W. B. Russel, Physics Today 51, 24 (1998).

[37] B. J. Ackerson and P. N. Pusey, Phys. Rev. Lett. 61, 1033 (1988).

[38] P. N. Pusey, J. Phys.: Condens. Matter 20, 494202 (2008). 
[39] S. Martin, G. Bryant, and W. van Megen, Phys. Rev. E 67, 061405 (2003).

[40] M. Cloitre, R. Borrega, and L. Leibler, Phys. Rev. Lett. 85, 4819 (2000).

[41] P. N. Pusey, E. Zaccarelli, C. Valeriani, E. Sanz, W. C. K. Poon, and M. E. Cates, Philos. Trans. A Math. Phys. Eng. Sci. 367, 4993 (2009).

[42] H. Löwen, T. Palberg, and R. Simon, Phys. Rev. Lett. 70, 1557 (1993).

[43] H. Löwen and G. Szamel, J. Phys.: Condens. Matter 5, 2295 (1993).

[44] W. Lechner and C. Dellago, J. Chem. Phys 129, 114707 (2008).

[45] P. J. Steinhardt, D. R. Nelson, and M. Ronchetti, Phys. Rev. B, PRB 28, 784 (1983).

[46] K. Kratzer and A. Arnold, Soft Matter 11, 2174 (2015).

[47] J. Hilhorst, J. R. Wolters, and A. V. Petukhov, Cryst. Eng. Comm. 12, 3820 (2010).

[48] F. W. Billmeyer, Textbook of Polymer Science (Wiley-Interscience, 1971). 